\title{
Diversidad de aves de hábitats naturales y modificados en un paisaje de la Depresión Central de Chiapas, México
}

\section{Jorge E. Ramírez-Albores}

1. Facultad de Estudios Superiores Zaragoza, Universidad Nacional Autónoma de México. México, D. F. Dirección actual: Manuel Bonilla \# 357, Manzana 44, Col. Santa Martha Acatitla, C. P. 09510, Delegación Iztapalapa. México, D.F.; jorgeramirez22@hotmail.com

\author{
Recibido 13-V-2008. Corregido 10-VII-2009. Aceptado 06-VIII-2009.
}

\begin{abstract}
Bird biodiversity in natural and modified habitats in a landscape of the Central Depression of Chiapas, Mexico. In many parts of the neotropics, the original habitats are rapidly changing because of excessive logging, agriculture and livestock activity, with an often negative impact on bird communities. I present an analysis of the diversity and richness of birds in a fragmented landscape of the Central Chiapas Depression. Fieldwork was conducted from February 2003 to January 2004. Using point counts, a total of 35 families and 225 bird species were registered (164 residents and 61 migratory); 3\% are abundant and 30\% rare. Diversity, species richness and number of individuals were significantly higher in tropical deciduous forest $\left(\mathrm{H}^{\prime}=3.41,178\right.$ species ANOVA $\left.p<0.0001\right)$, which also had the greatest number of species restricted to a single vegetation type ( 39 species). The incorporation and maintenance of natural and modified habitats are necessary for the survival and reproduction of many birds species in the study area. Rev. Biol. Trop. 58 (1): 511-528. Epub 2010 March 01.
\end{abstract}

Key words: richness, birds, tropical deciduous forest, tropical semideciduous forest, secondary forest, tropical oak forest, gallery forest, live fences, agricultural fields, cattle pastures, Chiapas.

En muchas partes de los neotrópicos, los hábitats originales están siendo rápidamente modificados debido a la tala excesiva, la agricultura y la ganadería. Muchos de los fragmentos de vegetación nativa están dominados por pastizales y potreros, y terrenos dedicados a la agricultura. Esta transformación del hábitat original ha tenido un impacto negativo sobre las comunidades de aves y otros grupos faunísticos, reduciendo la biodiversidad y la cantidad del hábitat original, interrumpiendo procesos ecológicos y modificando su composición (Dirzo \& García 1992, Daily et al. 2001).

Para el caso particular de las aves, varios estudios han demostrado que la transformación del hábitat original hacia pastizales y/o zonas agrícolas ha afectado negativamente a la comunidad de aves, modificando su riqueza, diversidad, composición y reduciendo el tamaño poblacional de algunas especies (Rappole \& Morton 1985, Kricher \& Davis 1989, Laurance \& Bierregaard 1997, Renjifo 1999). Estudios más recientes han logrado documentar que el área y su grado de aislamiento son determinantes del número de especies que un hábitat puede mantener. Por ejemplo, Loman \& Von Schantz (1991) reportan que en hábitats originales aislados, pequeños y cercanos a otros se presenta una alta diversidad y densidad de aves, debido a que las especies de estos utilizan los recursos de los hábitats originales que los rodean. Warburton (1997) encontró que la avifauna de hábitats originales fragmentados tienden a converger en su composición y están dominados generalmente por especies generalistas y tolerantes a la matriz. 
La Depresión Central de Chiapas, se caracteriza por ser el punto de contacto de varios hábitats (e. g., bosque tropical caducifolio, bosque tropical mediano y pequeños manchones de bosque de encino), la convergencia biogeográfica de dos rutas migratorias importantes (del Golfo y del Pacífico), y su heterogeneidad fisiográfica, que favorece el desarrollo de microambientes que inciden en la biodiversidad (Arriaga et al. 2000) y en la presencia de un número significativo de endemismos (Escalante et al. 1993). Los principales problemas ambientales que enfrenta esta región son la expansión de la frontera agropecuaria, los incendios forestales, el crecimiento demográfico, la extracción de leña, la contaminación de cuerpos y corrientes de agua y la ganadería, lo que conlleva a la pérdida de la biodiversidad presente en esta área (Reyes \& Souza 1997, Arriaga et al. 2000). Dentro de esta región, los estudios que se han realizado sobre su avifauna son escasos (e. g., Ovando 1990, Fernández 2002, Ramírez-Albores 2004). En el presente trabajo se caracteriza la avifauna presente en un mosaico de hábitats originales y modificados en el municipio de San Fernando, Chiapas, México. En el trabajo se describe el estatus migratorio y abundancia relativa de las especies de aves de los diferentes hábitats presentes en el área de estudio.

\section{MATERIAL Y MÉTODOS}

El trabajo de campo se llevó a cabo en el municipio de San Fernando, Chiapas $\left(258.3 \mathrm{Km}^{2}, 16^{\circ} 52^{\prime} \mathrm{N}\right.$ y $93^{\circ} 12^{\prime} \mathrm{W}$; Fig. 1). El área presenta una altitud de 900 m.s.n.m. y se localiza en la región fisiográfica denominada Depresión Central de Chiapas. El clima va de cálido subhúmedo, con lluvias en verano

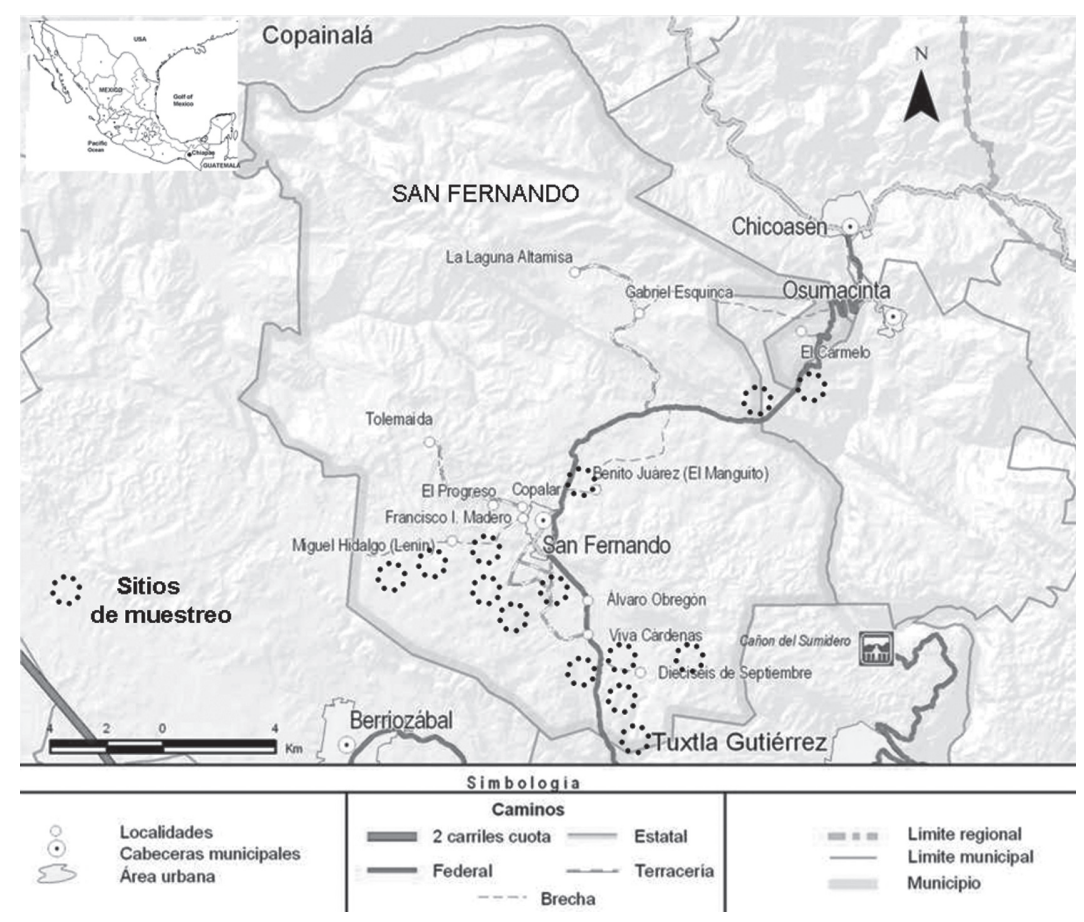

Fig. 1. Área de estudio y de los sitios de muestreo en Chiapas, México. Los círculos (•) son algunas de las localidades más representativas en el área de estudio.

Fig. 1. Geographical location of the study area and the sampling sites in Chiapas, Mexico. Circles (•) are some of the most representative locations in the study area. 
(de mayo a octubre, siendo mayo el mes más caluroso), a semicálido subhúmedo en altitudes superiores a los 1000m.s.n.m. (FORTAM 1984). La temperatura media anual es de $22^{\circ} \mathrm{C}$ y la precipitación anual varía entre 500 a $2500 \mathrm{~mm}$. La vegetación dominante es el bosque tropical caducifolio, así como una mezcla de manchones de bosque tropical mediano, encinar tropical y bosque de galería, además, de zonas con distinto grado de sucesión vegetal, cultivos, pastizales y zona suburbana (Miranda 1975, Reyes \& Souza 1997).

Para el registro de la avifauna presente en el área de estudio se consideraron los hábitats dominantes en el paisaje (Fig. 1) que fueron seleccionados con base en un análisis de fotografías aéreas (escala 1:75.000; INEGI 2001) y su posterior corroboración en campo.

El bosque tropical caducifolio tiene una superficie aproximada de 245 ha. Presenta dos estratos arbóreos, además de un arbustivo y un herbáceo. La altura de la vegetación es de 10 a $12 \mathrm{~m}$ y esta compuesta principalmente por Bursera simaruba, B. bipinnata, Casearia sp., Ceiba sp., Guettarda sp., Ardisia sp., Alvaradoa amorphoides y Jaquinia laurantifolia.

El bosque tropical mediano se desarrolla en lugares donde las condiciones son más húmedas principalmente en los fondos de cañadas y en suelos de mayor profundidad que el bosque tropical caducifolio y tiene una superficie aproximada de $72 \mathrm{ha}$. La altura varía entre 15 a $20 \mathrm{~m}$, los diámetros promedian $20 \mathrm{~cm}$. Las principales especies en este hábitat son Leacorea resoluta, Guettarda combsii, Bursera simaruba, B. bipinnata, Ficus cookii, Cedrela odorata y Daphnopsis mexicana.

La vegetación secundaria o acahual se divide en dos estratos y tiene una superficie de 214ha. El primero corresponde a una vegetación de tres a séis años de edad. Está caracterizado por un estrato arbustivo con una altura de $1 \mathrm{a} 2 \mathrm{~m}$, compuesto principalmente por especies pioneras como gramíneas y leguminosas. El segundo es una etapa avanzada en la sucesión del bosque tropical caducifolio. Existe un dosel arbóreo bien definido y su composición refleja una mezcla de elementos secundarios como de especies de interior. Los diámetros promedian $10 \mathrm{~cm}$ y la altura sobrepasa los $10 \mathrm{~m}$. Las principales especies son Alvaradoa amorphoides, Bursera simaruba, Heliocarpus reticulatus, Acacia cornigera y Casearia sp.

Los cercos vivos son un tipo de vegetación que se establece para delimitar una parcela o una propiedad, utilizándose principalmente especies de crecimiento rápido y que toleran la poda periódica de sus ramas como Bursera simaruba, B. bipinnata, Jatropha curcas, Cordia alliodora, $C$. dentata y Tecota stans. Tiene una superficie de 32 ha.

El encinar tropical se desarrolla a partir de los $1000 \mathrm{msnm}$ en suelos arenosos y alcanza una altura de hasta $10 \mathrm{~m}$. Tiene una superficie de 114ha. Varias especies de Quercus forman parte del estrato arbóreo, principalmente Quercus peduncularis, $Q$. polymorpha y $Q$. conspersa.

El bosque de galería se establece en los márgenes de los ríos y arroyos. El estrato arbóreo alcanza una altura de hasta $20 \mathrm{~m}$ y esta caracterizado por Zuelania guidonia, Daphnopsis mexicana, Pithecellobium lanceolatum, Trichilia hyrta y Diospyros sp. Tiene una superficie de 23ha.

Los potreros/pastizales están caracterizados por la presencia de Acacia pennatula, A. cornigera y Guazuma ulmifolia. Tiene una superficie de 288ha. Las zonas de cultivo son principalmente de maíz, y tiene una superficie de 617ha.

El trabajo de campo comprendió 156 días, de muestreos mensuales de febrero de 2003 a enero de 2004. Se empleó el método de conteo por parcelas con radio fijo (Hutto et al.1986, Ralph et al. 1995) donde se registraron todas las especies de aves observadas y escuchadas durante 5 minutos dentro una circunferencia con radio de $25 \mathrm{~m}$ y con una distancia mínima aproximada de $100 \mathrm{~m}$ entre cada parcela (Hutto et al.1986). Cada hábitat $(\mathrm{n}=8$; bosque tropical caducifolio, bosque tropical mediano, vegetación secundaria, encinar tropical, bosque de galería, cercos vivos, cultivos, pastizales y/o potreros) y su réplica $(n=6)$ para un total de 48 réplicas, fueron visitados por lo menos una 
vez al mes y se realizaron 12 conteos por punto cada mes. Las parcelas se ubicaron al azar y el número de puntos por parcela (de cuatro a ocho puntos por parcela) dependió de la extensión de los diferentes hábitats de estudio.

Las especies de aves se determinaron de forma visual usando las guías de campo (Peterson \& Chalif 1989, Howell \& Webb 1995, National Geographic Society 1999) y auditiva, al cotejar los cantos con grabaciones de las mismas especies (Duncan 1993). La riqueza de especies de aves de cada hábitat se obtuvo sumando las especies observadas dentro de cada uno y en la periferia de estos. A cada especie se le asignó un estatus de permanencia en cada hábitat, ya sea residente, visitante de invierno, transitoria, residente de verano u ocasional de acuerdo con Howell \& Webb (1995). La nomenclatura taxonómica usada corresponde a la propuesta por la American Ornithologists' Union (2008).

La abundancia relativa se obtuvo dividiendo el número de individuos de la especie ${ }_{1}$ entre el número total de individuos, multiplicado por 100 para obtener el porcentaje $\left(P_{1}=n \mathrm{~s}_{1} /\right.$ $\Sigma n$, donde $P_{1}$ representa la abundancia relativa de la especie ${ }_{1}, n \mathrm{~s}_{1}$ el número de individuos de la especie ${ }_{1}, \Sigma n$ el número total de individuos). El criterio utilizado para indicar los valores de abundancia fue el de Pettingil (1969), quien con base en un porcentaje determina la categoría de abundancia de cada especie: abundante (90-100\%), común (65-89\%), moderadamente común (31-64\%), no común (10-30\%) y rara (1-9\%).

La dieta o subdieta de las especies de aves se determinó por medio de la revisión de trabajos previos o relacionados con estudios avifaunísticos y corroborada con las observaciones realizadas en campo.

La similitud en la composición de especies de aves entre hábitats se determinó con el índice de Sorensen (IS $=2 \mathrm{~S} / \mathrm{N}_{1}+\mathrm{N}_{2}$, donde $\mathrm{S}$ es el número de especies compartidas, $\mathrm{N}_{1}$ es el número de especies del hábitat $1, \mathrm{y} \mathrm{N}_{2}$ es el número de especies del hábitat 2; Ravinovich 1981). En cada hábitat se determino el índice de diversidad y equidad de Shannon $\left(\mathrm{H}^{\prime}\right)$. Se empleó un análisis de varianza (ANOVA) por medio del programa Statistica, para comparar la riqueza de especies, el número de individuos de las especies de aves y los valores de diversidad entre hábitats. La prueba de comparaciones múltiples de Tukey fue usada para comparaciones post-hoc entre hábitats (Zar 1996).

\section{RESULTADOS}

Composición general: Se registró un total de 8414 individuos de aves pertenecientes a 35 familias y a 225 especies, que fueron registradas en 1092 horas de observación (Anexo 1). Las familias con mayor número de especies fueron Tyrannidae con 30, Parulidae con 30, Icteridae con 16 y Trochilidae con 14 (Anexo 1).

Del total de especies registradas, 164 fueron residentes, 45 visitantes de invierno, 15 transitorias y una residente de verano. Ocho especies son consideradas como abundantes, 30 como comunes, 57 como escasas, 61 como irregulares y 69 como raras. En cuanto a la dieta o subdieta de las especies, 64 fueron insectívoras, 65 insectívora/frugívoras, 24 carnívoras, 18 granívora/frugívoras, 14 nectarívoras y 12 granívoras (Anexo 1). El mayor número de especies se registró en el bosque tropical caducifolio (178), 128 en vegetación secundaria, 76 en cercos vivos, y 69 en el bosque tropical mediano (Anexo 1).

Del total de especies registradas, 10 son endémicas o cuasiendémicas a México (Aratinga holochlora, Amazilia yucatanensis, A. viridifrons, Momotus mexicanus, Uropsila leucogastra, Basileupterus rufifrons, Granatellus venustus, Arremonops rufivirgatus, Pheucticus chrysopeplus, Cacicus melanicterus; GonzálezGarcía \& Gómez de Silva 2003), y 28 se encuentran enlistadas en la NOM-059-SEMARNAT-2001 (SEMARNAT 2002; Anexo 1).

Comparación entre hábitats: La riqueza de especies varió significativamente entre los hábitats, siendo el bosque tropical caducifolio (178 especies) y la vegetación secundaria (128 especies) los que presentaron la mayor riqueza, mientras que el encinar tropical (45 especies) y 
CUADRO 1

Comparación de la diversidad y riqueza de aves entre los diferentes hábitats en el área de estudio

TABLE 1

Comparison of the diversity and richness of birds among different habitats in the study area

$\begin{array}{lcccccccc} & \text { BTC } & \text { BTM } & \text { BE } & \text { Hábitats } & \text { BG } & \text { Pp } & \text { C } & \text { Cv } \\ \text { \# especies } & 178 & 69 & 45 & 128 & 36 & 51 & 54 & 76 \\ \text { \# individuos promedio } & 90 & 58 & 32 & 81 & 22 & 31 & 33 & 44 \\ \text { Índice de Shannon (H') } & 3.39 & 3.24 & 3.03 & 3.16 & 3.03 & 3.01 & 3.02 & 3.12 \\ \text { Equidad (E') } & 0.88 & 0.84 & 0.81 & 0.85 & 0.81 & 0.80 & 0.80 & 0.84\end{array}$

* BTC (bosque tropical caducifolio), Pp (pastizal/potrero), Cv (cercos vivos), BG (bosque de galería), BTM (bosque tropical mediano), Vs (vegetación secundaria), C (cultivos), BE (encinar tropical).

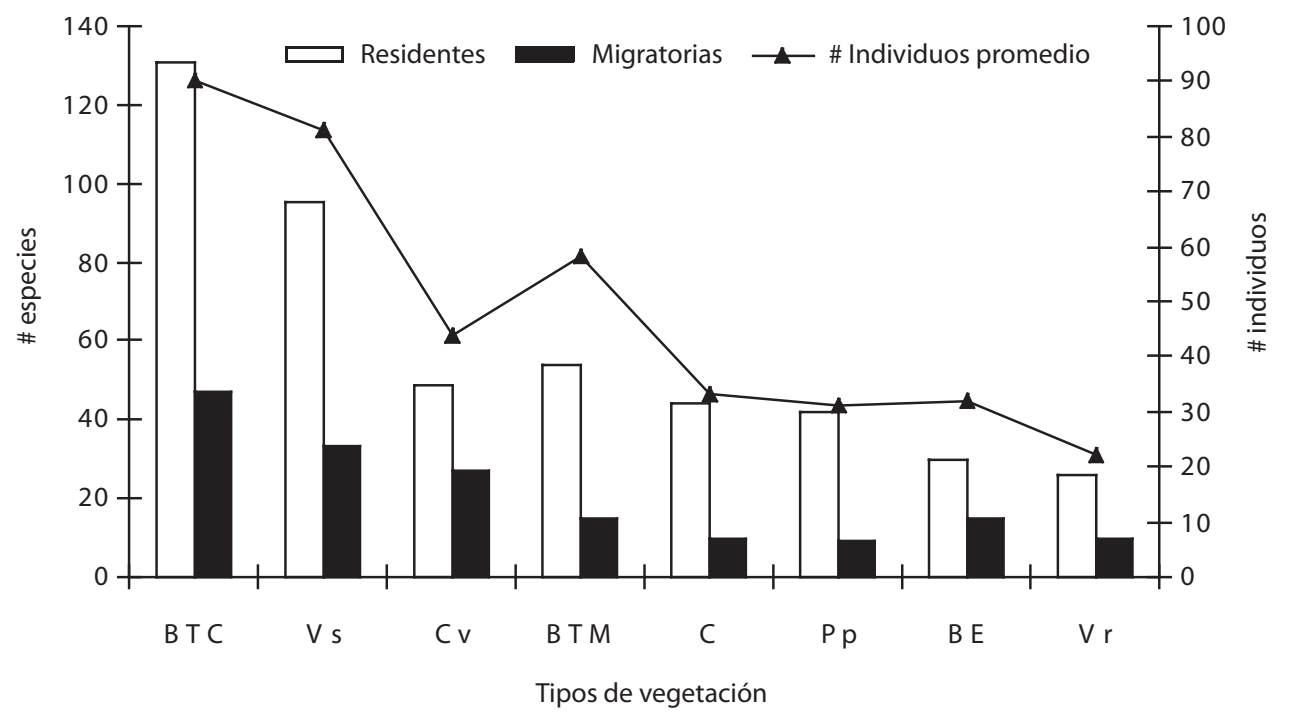

Fig. 2. Riqueza de especies y número de individuos en los diferentes hábitats en el área de estudio. Las medias no fueron estadísticamente diferentes (prueba de comparaciones múltiples de Tukey post-hoc, $\mathrm{p}<0.1$ )

Fig. 2. Species richness and number of individuals in different habitats in the study area. (Tukey post-hoc multiple comparison test, $\mathrm{p}<0.1)$.

el bosque de galería (36 especies) registraron los menores valores $\left(\mathrm{F}_{7.66}=75.3, \mathrm{P}<0.0001\right.$; Cuadro 1, Fig. 2). Para los valores de diversidad de especies varió significativamente entre los hábitats $\left(\mathrm{F}_{7.66}=124.8, \mathrm{P}<0.0001\right)$, siendo el bosque tropical caducifolio $\left(\mathrm{H}^{\prime}=3.39\right)$ y el bosque tropical mediano $\left(\mathrm{H}^{\prime}=3.34\right)$ los que presentaron los mayores valores de diversidad, mientras que los hábitats perturbados (i.e., cultivos con
$\mathrm{H}^{\prime}=3.02$ y potreros/pastizales con $\mathrm{H}^{\prime}=3.01$ ) registraron los menores valores (Cuadro 2, Fig. 2). En cuanto al número de individuos también se presentaron diferencias significativas entre los hábitats $\left(\mathrm{F}_{7.66}=114.2, \mathrm{P}<0.0001\right)$, el mayor número de individuos promedio se registró en el bosque tropical caducifolio (90) y vegetación secundaria (81), mientras que los menores valores se presentaron en el potrero/pastizal 
CUADRO 2

Matriz de valores de similitud de especies de aves entre hábitats

TABLE 2

Matrix similarity of bird species among hábitats

$\begin{array}{lccccccc} & \text { BTM } & \text { BE } & \text { Vs } & \text { BG } & \text { Pp } & \text { C } & \text { Cv } \\ \text { BTC } & 0.49 & 0.37 & 0.72 & 0.31 & 0.19 & 0.21 & 0.50 \\ \text { BTM } & & 0.44 & 0.38 & 0.44 & 0.16 & 0.14 & 0.32 \\ \text { BE } & & & 0.45 & 0.57 & 0.31 & 0.22 & 0.53 \\ \text { Vs } & & & & 0.34 & 0.27 & 0.32 & 0.58 \\ \text { BG } & & & & & 0.20 & 0.11 & 0.44 \\ \text { Pp } & & & & & & 0.76 & 0.27 \\ \text { C } & & & & & & & 0.20\end{array}$

* BTC (bosque tropical caducifolio), Pp (pastizal/potrero), $\mathrm{Cv}$ (cercos vivos), BG (bosque de galería), BTM (bosque tropical mediano), Vs (vegetación secundaria), C (cultivoS), BE (encinar tropical).
(31) y en el bosque de galería (22; Cuadro 2, Fig. 2).

Con respecto al estatus migratorio, el mayor número de especies residentes y migratorias se registró en el bosque tropical caducifolio (131 residentes y 47 migratorias) y vegetación secundaria (95 residentes y 33 migratorias), mientras que el menor número se registró en el encinar tropical (30) y bosque de galería (26) en cuanto a residentes, y bosque de galería (10) y pastizal/potreros (9) en cuanto a migratorias, encontrando diferencias significativas $\left(\mathrm{F}_{7.66}=85.8, \mathrm{P}<0.0001 ; \mathrm{F}_{7.66}=45.6\right.$, $p<0.0001$, respectivamente; Cuadro 3). Para la abundancia relativa, el mayor número de especies raras se registró en el bosque tropical caducifolio (47), en comparación con el encinar tropical donde se registraron dos especies

CUADRO 3

Número de especies de aves por hábitat, de acuerdo a su estatus migratorio, abundancia y hábitos alimenticios en el área de estudio

TABLE 3

Number of bird species by hábitat, according to their migratory status, abundance and diet habits in the study area

\begin{tabular}{|c|c|c|c|c|c|c|c|c|}
\hline & BTC & BTM & $\mathrm{BE}$ & Hábitats Vs & BG & $\mathrm{Pp}$ & $\mathrm{C}$ & $\mathrm{Cv}$ \\
\hline \multicolumn{9}{|l|}{ Estatus migratorio } \\
\hline Residente & 131 & 54 & 30 & 95 & 26 & 42 & 44 & 49 \\
\hline Migratoria & 47 & 15 & 15 & 33 & 10 & 9 & 10 & 27 \\
\hline \multicolumn{9}{|l|}{ Abundancia } \\
\hline Rara & 47 & 15 & 2 & 25 & 5 & 13 & 11 & 13 \\
\hline Irregular & 52 & 14 & 4 & 36 & 4 & 7 & 8 & 20 \\
\hline Escasa & 48 & 20 & 18 & 36 & 10 & 16 & 18 & 22 \\
\hline Común & 24 & 14 & 14 & 23 & 12 & 11 & 11 & 18 \\
\hline Abundante & 7 & 6 & 7 & 8 & 5 & 4 & 6 & 3 \\
\hline \multicolumn{9}{|l|}{ Dieta/subdieta } \\
\hline Carnívora & 16 & 9 & 5 & 12 & 3 & 16 & 16 & - \\
\hline Omnívora & 6 & 5 & 4 & 4 & 2 & 1 & 2 & 3 \\
\hline Insectívora & 54 & 13 & 7 & 32 & 8 & 7 & 6 & 22 \\
\hline Insectívora-frugívora & 57 & 21 & 22 & 42 & 14 & 6 & 2 & 40 \\
\hline Nectarívora & 14 & 4 & - & 9 & 2 & - & - & 2 \\
\hline Granívora & 2 & - & 1 & 6 & - & 10 & 11 & - \\
\hline Granívora-insectívora & 2 & 1 & - & 2 & - & 5 & 6 & 2 \\
\hline Carnívora-insectívora & 3 & 2 & - & 2 & 1 & 1 & 1 & - \\
\hline Granívora-frugívora & 15 & 8 & 2 & 13 & 1 & 1 & 6 & 1 \\
\hline Carnívora-insectívora-frugívora & 6 & 4 & 2 & 3 & 3 & 1 & 1 & 2 \\
\hline Granívora-insectívora-frugívora & 2 & 1 & 2 & 3 & 1 & 3 & 3 & 3 \\
\hline
\end{tabular}


(Cuadro 3). Las especies abundantes estuvieron mejor representadas en la vegetación secundaria (8), y el menor número de especies se registró en los pastizales/potreros (4) y cercos vivos (3), presentando diferencias significativas entre los hábitats $\left(\mathrm{F}_{7.66}=91.3, \mathrm{P}<0.0001\right.$; Cuadro 3). En los gremios alimenticios hubo también diferencias significativas entre los hábitats (Cuadro 3). Las especies de aves carnívoras estuvieron mejor representadas en el bosque tropical caducifolio, cultivos y pastizal/ potrero (16 especies en cada uno), mientras los números más bajos se presentaron en el bosque de galería (3) y no se registró ninguna especie en los cercos vivos $\left(\mathrm{F}_{7.66}=56.5, p<0.0001\right)$. Las especies insectívoro-frugívoras fueron más abundantes en el bosque tropical caducifolio (57) que en los cultivos (2) y potreros/pastizales $\left(6 ; \mathrm{F}_{7.66}=39.6, p<0.0001\right)$. Las especies de aves granívoro-frugívoras estuvieron mejor representadas en el bosque tropical caducifolio (15) y vegetación secundaria (13), el número más bajo se registró en los cercos vivos, bosque de galería y potreros/pastizales con una especie cada uno, presentando diferencias significativas entre los hábitats $\left(\mathrm{F}_{7.66}=49.3, p<0.0001\right)$. El bosque tropical caducifolio (54) y la vegetación secundaria (32) presentaron un mayor número de especies insectívoras que el encinar tropical (7), potreros/pastizal (7) y cultivos $\left(6 ; \mathrm{F}_{7.66}=83.9, p<0.0001\right)$. El bosque tropical caducifolio presentó el mayor número de nectarívoros (14), en comparación con el encinar tropical, potreros/pastizales y cultivos donde no hubo registros de estas especies, presentando diferencias significativas entre los hábitats $\left(\mathrm{F}_{7.66}=40.0, p<0.0001\right)$.

En cuanto a la similitud en la composición de especies en los diferentes hábitats, los potreros/pastizales y las zonas de cultivo compartieron un mayor número de especies (IS=0.76), seguidos por el bosque tropical caducifolio y la vegetación secundaria (IS $=0.72$; Cuadro 2). Los valores más bajos de similitud fueron compartidos entre el bosque de galería y las zonas de cultivo (IS $=0.11$ ), y el bosque tropical mediano con zonas de cultivo y potreros/pastizales (IS $=0.14$ y IS $=0.16$, respectivamente;
Cuadro 2). Veinticuatro especies se registraron exclusivamente en el bosque tropical caducifolio, cuatro en el bosque tropical mediano (Microcerculus marginatus, Hylocichla mustelina, Dendroica coronata y Helmitheros vermivorum), dos especies en el bosque de galería (Megaceryle alcyon y Chloroceryle amazona), una especie en la vegetación secundaria (Picoides scalaris), una especie en la zona de potreros/pastizales (Bubulcus ibis).

\section{DISCUSIÓN}

La riqueza de especies de aves registrada en este estudio representa el $34 \%$ de las especies reportadas para Chiapas por Álvarez del Toro (1980) y Palomera et al. (1994). Esta alta riqueza puede deberse a que existen una gran variedad de hábitats, la convergencia biogeográfica de dos rutas migratorias importantes (del Golfo y del Pacífico) y a la heterogeneidad fisiográfica en el área de estudio (Arriaga et al. 2000).

En cuanto al estatus migratorio se observó que existe una mayor proporción de especies residentes $(73 \%)$ que migratorias $(27 \%$, visitantes de invierno, transitorias y residentes de verano), lo cual coincide con otras regiones tropicales (i.e., Karr 1990, Ortiz-Pulido et al. 1995, Almazán \& Navarro 2006, RamírezAlbores 2006). Karr et al. (1982) mencionan que en algunos ambientes tropicales las especies migratorias son capaces de producir cambios en la composición de las comunidades de aves. En este estudio las especies migratorias jugaron un papel menor en los cambios observados en la comunidad de aves.

Con respecto a la abundancia relativa de las especies, es notorio que una gran proporción de especies son consideradas como raras (30\%). La ausencia de un mayor número de registros de estas especies puede explicarse porque la región estudiada no es muy extensa $\left(258.3 \mathrm{Km}^{2}\right)$ y porque los cambios en el paisaje afectan particularmente a estas especies. De acuerdo con Arizmendi et al. (1990), es posible que en el área de estudio los movimientos altitudinales y latitudinales estén correlacionados 
con las fluctuaciones en la abundancia de las especies. Por ejemplo, en el área de estudio algunas de las especies consideradas como raras son claramente características de zonas templadas ( $>1500$ m.s.n.m.), cuyas poblaciones se dispersan a otras áreas durante la época de escasez de recursos (e.g., Aulacorhynchus prasinus, Vireo leucophrys), o son migratorias de paso u ocasionales (e.g., Archilochus colubris, Catharus minimus, Dendroica townsendi).

La composición de la avifauna en función de los gremios alimenticios está relacionada con la estructura de la vegetación (Laurance \& Bierregaard 1997). En los ambientes tropicales, los hábitats modificados son muy importantes para una gran cantidad de especies carnívoras, granívoras e insectívoras ya que de forma temporal o permanente proveen de dichos recursos dependiendo de su fenología y estacionalidad (Loiselle \& Blake 1994). Por otro lado, los hábitats con una estructura de vegetación más compleja y formada por varios estratos de cobertura se presentan principalmente especies de hábitos insectívoros, frugívoros y nectarívoros (Rappole et al. 1993). Los resultados en este estudio, concuerdan con lo antes mencionado, ya que los hábitats modificados (i.e., cultivos, potreros/pastizales) presentaron una proporción mayor de especies e individuos de hábitos carnívoros y granívoros comparados con los hábitats originales (i.e., bosque tropical caducifolio y bosque tropical mediano).

De forma general, la variedad de hábitats que presenta el área de estudio parece contribuir a la alta riqueza relativa de especies, especialmente considerando el número de especies que ocurren en el bosque tropical caducifolio (178). Esto puede ser debido a la complejidad estructural que lo hace uno de los ecosistemas con mayor riqueza de especies en México (Ceballos \& García 1995), y el hecho de poseer una mayor estratificación tanto horizontal como vertical con respecto a otros, lo que genera una mayor disponibilidad de hábitats y nichos ecológicos (Blake \& Loiselle 1991, McIntyre 1995, Villard et al. 1999), ya que la estructura vegetal determina la cantidad y distribución de los recursos que utilizan las aves.
Las diferencias en la diversidad y riqueza encontradas, indican que el bosque tropical caducifolio, el bosque tropical mediano y la vegetación secundaria presentan una diversidad y riqueza mayor en comparación con los otros hábitats modificados (e.g., cultivos, potreros/ pastizales). Lo anterior, coincide con otros estudios realizados en ambientes tropicales y que indican que la pérdida de hábitats originales influye directamente en la presencia, abundancia y persistencia de especies (Kattan et al. 1994, Laurance \& Bierregaard 1997). Los resultados obtenidos en este estudio sugieren que la riqueza y diversidad de especies varía entre los hábitats de estudio. Los hábitats naturales (bosque tropical caducifolio, bosque tropical mediano, vegetación secundaria) parecen ser atractivos para un mayor número de especies de aves, ya que tanto la riqueza como la diversidad fueron más altas en estos; lo que concuerda con otros estudios (Estrada et al. 1997, Petit et al. 1999, Blake \& Loiselle 2001, Bojorges \& López-Mata 2005). Los hábitats modificados (cultivos, cercos vivos, pastizales/potreros) tuvieron una importante contribución a la riqueza de especies de aves en el área de estudio. Estos hábitats proporcionan sitios de percha y recursos alimenticios (Lynch 1989). Lo anterior concuerda con lo reportado por Estrada et al. (1997), el cual reporta una riqueza de 226 en la región de Los Tuxtlas (Veracruz, México), encontrando el 79\% de las especies detectadas en zonas de bosque, $80 \%$ en campos agrícolas, $43 \%$ en cercos vivos y solo el $5 \%$ en pastizales/potreros. Por otra parte, Petit et al. (1999) en área de la parte central de Panamá encontraron que la riqueza de aves en hábitats modificados (i.e., plantaciones de café con sombra, áreas residenciales, pastizales y plantaciones de pino) es igual o similar a la de hábitats naturales.

Sin embargo, los cercos vivos concentraron la mayor riqueza de especies (76 especies) entre los hábitats modificados (e.g., cultivos, potreros/pastizales), probablemente debido a que su estructura vegetal es más compleja y diversa. Esto concuerda con otros estudios (i.e., Villaseñor 1993, Villaseñor \& Hutto 1995, 
Morales 2002), los cuales mencionan que los cercos vivos suelen ser muy atractivos para una gran cantidad de individuos y especies de aves, y además, pueden soportar altas densidades, ya que proveen recursos alimenticios, sitios de percha y refugio (Villaseñor \& Hutto 1995). Por ejemplo, en el área de estudio algunas especies de aves (i.e., Calocitta formosa, Cyanocorax yncas, Thryothorus pleurustictus) utilizan preferentemente corredores o cercos vivos en lugar de áreas abiertas o desmontadas (Wegner \& Merriam 1979) y las tasas de movimiento son significativamente más frecuentes a lo largo de corredores conectados con hábitats originales o con otros corredores (Hass 1995, Machtans et al. 1996). En el caso donde se conserva el hábitat original, la complejidad de la vegetación proporciona sitios alternos para algunas especies, compensando parcialmente la fragmentación y permitiendo la persistencia de especies residentes y migratorias (Morales 2002).

La similitud entre los hábitats de estudio indica la existencia de un alto intercambio de especies, así como de una aparente alta conectividad entre estos. Lo que sugiere que tanto la configuración del ambiente (i.e., paisaje, hábitat y microhábitat) como la cantidad disponible de éstos, no serían igualmente importantes en la distribución de las especies (Karr 1990) y podría conferir cambios en la composición de la comunidad de aves (Blake \& Loiselle 2001).

Aún cuando la conservación de las especies de aves depende de la comprensión clara de sus requerimientos de hábitat y de los procesos físicos y bióticos que lo mantienen (Askins 2000), se ha establecido que la combinación de hábitats naturales y modificados conduce a nuevas posibilidades de explotación diferencial del espacio (Willson 1974) y que la diversidad de especies de aves está vinculada a la diversidad del paisaje, por lo que la conservación de este último aseguraría la conservación de la diversidad de especies (Bôhning-Gaese 1997). Ante la continua fragmentación de los hábitats naturales y de acuerdo a los resultados de este estudio, la incorporación y mantenimiento de hábitats naturales y modificados son necesarios para la supervivencia y reproducción de muchas especies de aves en el área de estudio. El área de estudio, como muchas otras regiones del país, está siendo afectada por diversos factores antropogénicos, en especial por la expansión de la frontera agropecuaria, los incendios forestales, el crecimiento demográfico y la ganadería, lo cual incide directamente en las poblaciones de fauna silvestre. Los estudios de diversidad y riqueza de especies son aproximaciones que representan la información base para posteriormente evaluar mediante el monitoreo, los cambios asociados a distintos factores ambientales y especialmente los antropogénicos. El inventario aquí presentado podría ser considerado representativo para el área de estudio y puede servir de referencia para futuras investigaciones que se realicen en dicha región, además, que los esfuerzos de conservación deben enfocarse primero en los hábitats naturales que se mantienen en el paisaje y al mismo tiempo tratar de aumentar la cobertura arbórea de los hábitats modificados.

\section{AGRADECIMIENTOS}

Agradezco a El Colegio de la Frontera Sur (San Cristóbal) y al Consejo Nacional de Ciencia y Tecnología (CONACyT) por las facilidades para esta investigación y el apoyo financiero. A J.L. Rangel-Salazar, M.A. Martínez-Morales y a G. Escalona-Segura por sus comentarios y sugerencias durante la realización de la tesis. A todas las personas de las comunidades locales del municipio de San Fernando, Chiapas por su apoyo durante la realización del estudio. A los revisores anónimos por sus comentarios y sugerencias.

\section{RESUMEN}

Se presenta un análisis de la diversidad y riqueza de aves en un paisaje fragmentado en la Depresión Central de Chiapas. El trabajo de campo se realizó de febrero de 2003 a enero de 2004. Se utilizó el método de conteo por puntos, registrando un total 35 familias y 225 especies de aves. Del total de especies registradas 164 son residentes y 61 migratorias. El 3\% de las especies son abundantes y el 
$30 \%$ son raras. Se presentaron diferencias significativas en la diversidad, riqueza de especies y número de individuos entre los hábitats de estudio (ANOVA $p<0.0001$ ). La diversidad y riqueza de especies fue significativamente mayor en el bosque tropical caducifolio $\left(\mathrm{H}^{\prime}=3.41,178\right.$ especies), presentando también el mayor número de especies exclusivas (39). La incorporación y mantenimiento de hábitats naturales y modificados son necesarios para la supervivencia y reproducción de muchas especies de aves en el área de estudio.

Palabras clave: riqueza, aves, bosque tropical caducifolio, bosque tropical mediano, encinar tropical, vegetación secundaria, bosque de galería, cercos vivos, cultivos, pastizales y/o potreros, Chiapas.

\section{REFERENCIAS}

Almazán, N. R. C. \& A. G. Navarro. 2006. Avifauna de la subcuenca del río San Juan, Guerrero, México. Rev. Mex. Biodivers. 77: 103-114.

Álvarez del Toro, M. 1980. Las aves de Chiapas. Gobierno del Estado de Chiapas. Tuxtla Gutiérrez, Chiapas, México.

Arizmendi, M. C., H. Berlanga, L. Márquez-Valdelamar, L. Navarijo \& J. F. Ornelas. 1990. Avifauna de la región de Chamela, Jalisco. Cuadernos del Instituto de Biología No. 4, UNAM. México, D. F., México.

Arriaga, L., J. M. Espinoza, C. Aguilar, E. Martínez, L. Gómez, E. Loa \& J. Larson. 2000. Regiones prioritarias terrestres de México. CONABIO. México, D. F., México.

Askins, R. A. 2000. Restoring North America's birds. Yale. Nueva Haven, Connecticut, EEUU.

Blake, J. G. \& B. A. Loiselle. 1991. Variation in resource abundance effects capture rates of birds in three lowland habitats in Costa Rica. Auk 108: 114-130.

Blake, J. G. \& B. A. Loiselle. 2001. Birds assemblages in second-growth and old-growth forest, Costa Rica: perspectives from mist nest and point counts. Auk 118: 304-326.

Bôhning-Gaese, K. 1997. Determinants of avian species richness at different spatial scales. J. Biogeogr. 24: 49-60.

Bojorges, B. J. \& L. López-Mata. 2005. Riqueza y diversidad de especies de aves en una selva mediana subperennifolia en el centro de Veracruz, México. Acta Zool. Mex. 21:1-20.
Ceballos, G. \& A. García. 1995. Conserving neotropical biodiversity: the role of dry forest in western Mexico. Conserv. Biol. 9: 1349-1353.

Daily, G. C., P. Erhlich \& A. Azofeifa. 2001. Countryside biogeography: use of human-dominated habitats by the avifauna of the southern Costa Rica. Ecol. Appl. 11:1-13.

Dirzo, R. \& M. C. García. 1992. Rates of deforestation in Los Tuxtlas, a neotropical area in southeast Mexico. Conserv. Biol. 6: 84-90.

Duncan, C. D. 1993. Manual para el uso de la fonoteca de las aves de Chiapas "cassettes 1-8". PronaturaInstituto para la Ornitología del Campo, Universidad de Maine en Manchias, México.

Escalante, P., A. G. Navarro \& A. T. Peterson. 1993. A geographic, ecological, and historical analysis of land bird diversity in Mexico, p. 281-307. In T. P. Ramamorthy, R. Bye, A. Lot \& J. Fa (eds.). The biological diversity of Mexico: origins and distribution. Oxford, Nueva York, EEUU.

Estrada, A., R. Coates-Estrada \& D. A. Meritt. 1997. Anthropogenic landscape changes and avian diversity at Los Tuxtlas, Mexico. Biodivers. Conserv. 6: 19-43.

Fernández, M. Y. 2002. Áreas naturales en ciudades y su conservación: El caso de la avifauna de El Zapotal, Tuxtla Gutiérrez, Chiapas, México. Tesis de Maestría. El Colegio de la Frontera Sur. San Cristóbal de las Casas, Chiapas, México.

FORTAM. 1984. San Fernando, Diagnóstico Municipal. Gobierno Federal-Estatal-Municipal Plan Chiapas. Talleres Gráficos del Estado. Tuxtla Gutiérrez, Chiapas, México.

González-García, F. \& H. Gómez de Silva. 2003. Especies endémicas: riqueza, patrones de distribución y retos para su conservación. p. 150-194. In H. Gómez de Silva \& A. Oliveras de Ita (eds.). Conservación de aves. Experiencias en México. Cipamex-ConabioNational Fish and Wildlife Foundation. México, D.F.

Hass, C. A. 1995. Dispersal and use of corridors by birds in wooded patches on an agricultural landscape. Conserv. Biol. 9: 845-854.

Howell, S. N. \& S. Webb. 1995. A guide to the birds of Mexico and Northern Central America. Oxford, Nueva York, EEUU.

Hutto, R. L., S. M. Pletschet \& P. Hendricks. 1986. A fixed-radius point count method for non-breeding and breeding season use. Auk 103: 593-602. 
INEGI (Instituto Nacional de Estadística, Geografía e Informática). 2000. Fotografía aérea del municipio de San Fernando, Chiapas (escala 1:50.000). INEGI. México, D. F., México.

Karr, J. R. 1990. The avifauna of Barro Colorado Island and the Pipeline Roas, Panama, p. 183-198. In A. H. Gentry (ed.). Four neotropical rainforests. Yale, Nueva Haven, EEUU.

Karr, J. R., D. W. Schemske \& N. V. L. Brokaw. 1982. Temporal variation in the understory bird community of a tropical forest, p. 441-453. In E. G. Leigh Jr., A. S. Rand \& D. M. Windsor (eds.). The ecology of a tropical forest. Smithsonian Institution, Washington, D. C., EEUU.

Kattan, G. H., H. Álvarez-López \& M. Giraldo.1994. Forest fragmentation and bird extinction: San Antonio eighty years later. Conserv. Biol. 8: 138-146.

Kricher, J. C. \& W. E. Davis. 1989. Patterns of avian species richness in disturbed and undisturbed habitats in Belice, p. 240-246. In J. Hagan \& D. Johnston (eds.). Ecology and conservation of neotropical migrant landbirds. Smithsonian Institution, Washington, D. C., EEUU.

Laurance, W. F. \& R. O. Bierregaard, Jr. 1997. Tropical forest remnants: ecology, management, and conservation of fragmented communities. University of Chicago, Chicago, EEUU.

Loiselle, A. B. \& J. G. Blake. 1994. Annual variation in birds and plants of a tropical second-growth woodland. Condor 96: 368-380.

Loman, J. \& T. Von Schantz. 1991. Birds in a farmlandmore species in small than in large habitat island. Conserv. Biol. 5: 176-188.

Lynch, J. F. 1989. Distribution of overwintering neartic migrants in the Yucatan Peninsula, II: use of relative and human-modified vegetation. p. 179-196. In J. M. Hagan \& D. W. Johnston (eds.). Ecology and conservation of neotropical migrant landbirds. Smithsonian Institution, Washington, D. C., EEUU.

Machtans, C. S., M. Villard \& S. J. Hannon. 1996. Use of riparian buffer strips as movement corridors by forest birds. Conserv. Biol. 10: 1366-1379.

McIntyre, N. 1995. Effects on forest patch size on avian diversity. Landscape Ecol. 10: 85-99.
Miranda, F. 1975. La vegetación de Chiapas. Gobierno del Estado. Tuxtla Gutiérrez, Chiapas, México.

Morales, P. L. 2002. Efectos de la modificación del hábitat sobre la avifauna de la Reserva de la Biosfera Chamela-Cuixmala y sus alrededores. Tesis Licenciatura. Facultad de Ciencias, UNAM. México, D. F., México.

National Geographic Society. 1999. Field guide to the birds of North America. National Geographic Society. Washington, D. C., EEUU.

Ortiz-Pulido, R., H. Gómez de Silva, F. González-García \& A. Álvarez. 1995. Avifauna del Centro de Investigaciones Costeras La Mancha, Veracruz, México. Acta Zool. Mex. 66: 87-118.

Ovando D., L. 1990. Avifauna del Parque Nacional Cañón del Sumidero, Chiapas, México. Tesis de Licenciatura. Instituto de Ciencias y Artes de Chiapas. Tuxtla Gutiérrez, Chiapas, México.

Palomera-García, C., E. Santana \& R. Amparán-Salcido. 1994. Patrones de distribución de la avifauna en tres estados del occidente de México. An Inst Biol, UNAM, Serie Zoología 5: 137-175.

Peterson, R. T. \& E. L. Chalif. 1989. Aves de México: Guía de campo. Ed. Diana. México, D. F., México.

Petit, L. J., D. R. Petit, D. G. Christian \& H. D. W. Powell. 1999. Bird communities of natural and modified habitats in Panama. Ecography 22: 292-304.

Pettingill, O. S. Jr. 1969. Ornithology in laboratory and field. Burgués, Minneapolis, EEUU.

Ralph, C. J., J. R. Saber \& S. Droege. 1995. Monitoring bird populations by point counts. General Technical Report PSW-GTR-149. USDA Forest Service, Pacific Southwest Research Station. Albany, California, EEUU.

Ramírez-Albores, J. E. 2004. Efecto de la estructura del paisaje sobre la diversidad $\alpha, \beta$ y $\gamma$ de comunidades de aves en San Fernando, Chiapas, México. Tesis de Maestría, El Colegio de la Frontera Sur. San Cristóbal de las Casas, Chiapas, México.

Rappole, J. H., E. S. Morton, T. E. Lovejoy III \& J. R. Ruos. 1993. Aves migratorias neárticas en los neotrópicos. Conservation and Research Center. National Zoological Park. Front Royal, BC, Canadá. 
Rappole, J. H. \& E. S. Morton. 1985. Effects of habitat alteration on a tropical avian forest community, p. 1013-1021. In P. A. Buckley, E. S. Morton, R. Ridgely \& F. G. Buckley (eds.). Neotropical Ornithology. Ornithological Monographs 36.

Ravinovich, J.1981. Introducción a la ecología de poblaciones animales. Continental. México, D. F., México.

Renjifo, L. M. 1999. Composition changes in a subandean avifauna after long-term forest fragmentation. Conserv. Biol. 13: 1124-1139.

Reyes, A. \& M. Souza. 1997. Listados florísticos de México XVII: Depresión Central de Chiapas. La selva baja caducifolia. Instituto de Biología, UNAM. México, D. F., México.

SEMARNAT (Secretaría del Medio Ambiente y Recursos Naturales). 2002. Norma Oficial Mexicana NOM-059-SEMARNAT-2001. Protección ambiental-especies nativas de México de flora y fauna silvestres-categorías de riesgo y especificaciones para su inclusión, exclusión o cambio-lista de especies en riesgo. 6 de marzo de 2002 .

Villard M, M. K. Trzcinski \& G. Merriam. 1999. Fragmentation effects on forest birds: Relative influence of woodland cover and configuration on landscape occupancy. Conserv. Biol. 13: 774-783.

Villaseñor, J. F. 1993. The importance of agricultural border strips in the conservation of North American migratory land birds in western Mexico. M.Sc. thesis, University of Montana. Helena, EEUU.

Villaseñor, J. F. \& R. L. Hutto. 1995. The importance of agricultural areas for the conservation of neotropical migratory landbirds in Western Mexico, p 59-80. In M. H.

Wilson \& S. A. Sader (eds.). Conservation of neotropical migratory birds in Mexico. Miscellaneous Publication. Agricultural and Forest Experiment Station. Portland, Maine, EEUU.

Warburton, N. H. 1997. Structure and conservation of forest avifauna in isolated rain forest remnants in tropical Australia, p. 190-207. In W. F. Laurance \& R. O. Bierregaard, Jr. (eds.). Tropical forest remnants: ecology, management, and conservation of fragmented communities. Chicago, Chicago, EEUU.

Wegner, J. F. \& G. Merriam. 1979. Movements by birds and small mammals between a wood and adjoining farmland habitats. J. Appl. Ecol. 76: 27-40.

Willson, M. F. 1974. Avian community organization and habitat structure. Ecology 55: 1107-1029.

Zar, J. H. 1996. Biostatistical analysis. Prentice Hall. Nueva Jersey, EEUU.

\section{REFERENCIAS DE INTERNET}

American Ornithologists' Union. 2008. Check-list of North American birds. American Ornithologists' Union, Madison, Estados Unidos. (junio 3, 2009, http:// www.aou.org).

Ramírez-Albores, J. E. 2006. Variación en la composición de comunidades de aves en la Reserva de la Biosfera Montes Azules y áreas adyacentes, Chiapas, México. Biota Neotrop 6, (junio 3, 2009, http://www.biotaneotropica. org.br/v6n2/ pt/abstract?article+bn02806022006). 
ANEXO 1

Especies de aves del área de estudio en la Depresión Central de Chiapas, México

APPENDIX 1

Bird species in the study area, Central Depression of Chiapas, Mexico

\begin{tabular}{|c|c|c|c|c|c|c|c|c|c|c|c|c|}
\hline \multirow[b]{2}{*}{ Especies } & \multicolumn{8}{|c|}{ Hábitats } & \multirow{2}{*}{$\begin{array}{c}\text { Estatus } \\
\text { migratorio }\end{array}$} & \multirow[b]{2}{*}{ Dieta } & \multirow{2}{*}{$\begin{array}{c}\text { Abundancia } \\
\text { relativa }\end{array}$} & \multirow{2}{*}{$\begin{array}{c}\text { Estatus } \\
\text { en la } \\
\text { NOM-059 }\end{array}$} \\
\hline & BTC & BTM & Vs & $\mathrm{BE}$ & $\mathrm{Pp}$ & $\mathrm{C}$ & $\mathrm{CV}$ & BG & & & & \\
\hline Crypturellus soui & $\mathrm{x}$ & & & & & & & & $\mathrm{R}$ & $\mathrm{O}$ & $\mathrm{E}$ & $\operatorname{Pr}$ \\
\hline Crypturellus cinnamomeus & $\mathrm{x}$ & $\mathrm{x}$ & & & & & & & $\mathrm{R}$ & $\mathrm{O}$ & I & \\
\hline Ortalis vetula & $\mathrm{x}$ & $\mathrm{x}$ & $\mathrm{x}$ & $\mathrm{x}$ & & & & & $\mathrm{R}$ & GF & $\mathrm{C}$ & \\
\hline Penelope purpurascens & $\mathrm{x}$ & $\mathrm{x}$ & & & & & & & $\mathrm{R}$ & GF & $\mathrm{R}$ & A \\
\hline Colinus virginianus & & & & & $\mathrm{x}$ & $\mathrm{x}$ & & & $\mathrm{R}$ & $\mathrm{G}$ & $\mathrm{C}$ & \\
\hline Bubulcus ibis & & & & & $\mathrm{x}$ & & & & $\mathrm{R}$ & I & $\mathrm{C}$ & \\
\hline Coragyps atratus & $\mathrm{x}$ & $\mathrm{x}$ & $\mathrm{x}$ & $\mathrm{x}$ & $\mathrm{x}$ & $\mathrm{x}$ & & $\mathrm{x}$ & $\mathrm{R}$ & $\mathrm{C}$ & A & \\
\hline Cathartes aura & $\mathrm{x}$ & $\mathrm{x}$ & $\mathrm{x}$ & $\mathrm{x}$ & $\mathrm{x}$ & $\mathrm{x}$ & & $\mathrm{x}$ & $\mathrm{R}$ & $\mathrm{C}$ & A & \\
\hline Pandion haliaetus & $\mathrm{x}$ & $\mathrm{x}$ & $\mathrm{x}$ & & & & & & $\mathrm{T}$ & $\mathrm{C}$ & $\mathrm{R}$ & \\
\hline Elanus leucurus & & & & & $\mathrm{x}$ & $\mathrm{x}$ & & & $\mathrm{R}$ & $\mathrm{C}$ & $\mathrm{R}$ & \\
\hline Rosthramus sociabilis & & $\mathrm{x}$ & $\mathrm{x}$ & & & & & & $\mathrm{R}$ & $\mathrm{C}$ & $\mathrm{R}$ & $\operatorname{Pr}$ \\
\hline Ictinia mississippiensis & $\mathrm{x}$ & $\mathrm{x}$ & & $\mathrm{x}$ & $\mathrm{x}$ & $\mathrm{x}$ & & & $\mathrm{T}$ & $\mathrm{C}$ & I & $\operatorname{Pr}$ \\
\hline Accipiter cooperii & $\mathrm{x}$ & $\mathrm{x}$ & $\mathrm{x}$ & $\mathrm{x}$ & $\mathrm{x}$ & $\mathrm{x}$ & & & $\mathrm{W}$ & $\mathrm{C}$ & $\mathrm{E}$ & $\operatorname{Pr}$ \\
\hline Buteogallus anthracinus & $\mathrm{x}$ & & $\mathrm{x}$ & & $\mathrm{x}$ & $\mathrm{x}$ & & & $\mathrm{R}$ & $\mathrm{C}$ & $\mathrm{E}$ & $\operatorname{Pr}$ \\
\hline Buteo magnirostris & $\mathrm{x}$ & & $\mathrm{x}$ & $\mathrm{x}$ & $\mathrm{x}$ & $\mathrm{x}$ & & & $\mathrm{R}$ & $\mathrm{C}$ & $\mathrm{E}$ & \\
\hline Buteo nitidus & $\mathrm{x}$ & & $\mathrm{x}$ & & $\mathrm{x}$ & $\mathrm{x}$ & & & $\mathrm{R}$ & $\mathrm{C}$ & I & \\
\hline Buteo brachyurus & $\mathrm{x}$ & & $\mathrm{x}$ & & & & & & $\mathrm{R}$ & $\mathrm{C}$ & I & \\
\hline Buteo albicaudatus & $\mathrm{x}$ & $\mathrm{x}$ & & & & & & & $\mathrm{R}$ & $\mathrm{C}$ & I & $\operatorname{Pr}$ \\
\hline Buteo jamaicensis & $\mathrm{x}$ & & $\mathrm{x}$ & & $\mathrm{x}$ & $\mathrm{x}$ & & & $\mathrm{W}$ & $\mathrm{C}$ & E & \\
\hline Caracara cheriway & & & & & $\mathrm{x}$ & $\mathrm{x}$ & & & $\mathrm{R}$ & $\mathrm{C}$ & $\mathrm{R}$ & \\
\hline Herpetotheres cachinnans & & & & & $\mathrm{x}$ & $\mathrm{x}$ & & & $\mathrm{R}$ & $\mathrm{C}$ & I & \\
\hline Falco sparverius & & & & & $\mathrm{x}$ & $\mathrm{x}$ & & & $\mathrm{R}$ & $\mathrm{C}$ & $\mathrm{E}$ & \\
\hline Falco femoralis & & & & & $\mathrm{x}$ & $\mathrm{x}$ & & & $\mathrm{R}$ & $\mathrm{C}$ & $\mathrm{R}$ & \\
\hline Falco peregrinus & & & & & $\mathrm{x}$ & $\mathrm{x}$ & & & $\mathrm{W}$ & $\mathrm{C}$ & I & $\operatorname{Pr}$ \\
\hline Patagioenas flavirostris & $\mathrm{x}$ & & $\mathrm{x}$ & & & & & & $\mathrm{R}$ & GF & E & \\
\hline Patagioenas nigrirostris & $\mathrm{x}$ & & $\mathrm{x}$ & & & $\mathrm{x}$ & & & $\mathrm{R}$ & GF & I & \\
\hline Zenaida asiatica & $\mathrm{x}$ & & $\mathrm{x}$ & & & $\mathrm{x}$ & & & $\mathrm{R}$ & GF & A & \\
\hline Zenaida macroura & $\mathrm{x}$ & & $\mathrm{x}$ & & & $\mathrm{x}$ & & & W & GF & I & \\
\hline Columbina inca & & & $\mathrm{x}$ & $\mathrm{x}$ & $\mathrm{x}$ & $\mathrm{x}$ & & & $\mathrm{R}$ & G & A & \\
\hline Columbina passerina & & & $\mathrm{x}$ & & $\mathrm{x}$ & $\mathrm{x}$ & & & $\mathrm{R}$ & G & $\mathrm{E}$ & \\
\hline Columbina minuta & $\mathrm{x}$ & & $\mathrm{x}$ & & & & & $\mathrm{x}$ & $\mathrm{R}$ & G & $\mathrm{E}$ & \\
\hline Columbina tapalcoti & & & $\mathrm{x}$ & & $\mathrm{x}$ & $\mathrm{x}$ & & & $\mathrm{R}$ & $\mathrm{G}$ & $\mathrm{R}$ & \\
\hline Claravis pretiosa & $\mathrm{x}$ & & $\mathrm{x}$ & & & & & & $\mathrm{R}$ & GF & $\mathrm{R}$ & \\
\hline Leptotila verreauxi & $\mathrm{x}$ & $\mathrm{x}$ & $\mathrm{x}$ & & & $\mathrm{x}$ & & $\mathrm{x}$ & $\mathrm{R}$ & GF & $\mathrm{C}$ & \\
\hline Geotrygon montana & $\mathrm{x}$ & & $\mathrm{x}$ & & & & & & $\mathrm{R}$ & GF & $\mathrm{R}$ & \\
\hline Aratinga holochlora & $\mathrm{x}$ & & & & & & & & $\mathrm{R}$ & GF & $\mathrm{R}$ & $\operatorname{Pr}$ \\
\hline Aratinga nana & $\mathrm{x}$ & & $\mathrm{x}$ & & & $\mathrm{x}$ & & & $\mathrm{R}$ & GF & $\mathrm{C}$ & $\operatorname{Pr}$ \\
\hline Amazona albifrons & $\mathrm{x}$ & $\mathrm{x}$ & & & & & & & $\mathrm{R}$ & GF & $\mathrm{E}$ & \\
\hline Amazona autumnalis & $\mathrm{x}$ & $\mathrm{x}$ & & & & & & & $\mathrm{R}$ & GF & I & \\
\hline Piaya cayana & $\mathrm{x}$ & & $\mathrm{x}$ & & & & & & $\mathrm{R}$ & CIF & $\mathrm{C}$ & \\
\hline Coccyzus minor & $\mathrm{x}$ & & $\mathrm{x}$ & & & & & & $\mathrm{R}$ & $\mathrm{CI}$ & $\mathrm{E}$ & \\
\hline Tapera naevia & $\mathrm{x}$ & & & & & $\mathrm{x}$ & & & $\mathrm{R}$ & CIF & $\mathrm{E}$ & \\
\hline Dromococcyx phasianellus & $\mathrm{x}$ & $\mathrm{x}$ & & & & & & & $\mathrm{R}$ & $\mathrm{CI}$ & I & \\
\hline Morococcyx erythropygus & $\mathrm{x}$ & $\mathrm{x}$ & $\mathrm{x}$ & & & & & & $\mathrm{R}$ & CI & $\mathrm{E}$ & \\
\hline
\end{tabular}


ANEXO 1 (Continuación)

Especies de aves del área de estudio en la Depresión Central de Chiapas, México

APPENDIX 1 (Continued)

Bird species in the study area, Central Depression of Chiapas, Mexico

\begin{tabular}{|c|c|c|c|c|c|c|c|c|c|c|c|c|}
\hline \multirow[b]{2}{*}{ Especies } & \multicolumn{8}{|c|}{ Hábitats } & \multirow{2}{*}{$\begin{array}{l}\text { Estatus } \\
\text { migratorio }\end{array}$} & \multirow[b]{2}{*}{ Dieta } & \multirow{2}{*}{$\begin{array}{l}\text { Abundancia } \\
\text { relativa }\end{array}$} & \multirow{2}{*}{$\begin{array}{c}\text { Estatus } \\
\text { en la } \\
\text { NOM-059 }\end{array}$} \\
\hline & BTC & BTM & Vs & $\mathrm{BE}$ & $\mathrm{Pp}$ & $\mathrm{C}$ & $\mathrm{Cv}$ & BG & & & & \\
\hline Geococcyx velox & & & & & $\mathrm{x}$ & $\mathrm{x}$ & & & $\mathrm{R}$ & CI & $\mathrm{E}$ & \\
\hline Crotophaga sulcirostris & & & $\mathrm{x}$ & & $\mathrm{x}$ & $\mathrm{x}$ & $\mathrm{x}$ & & $\mathrm{R}$ & GIF & $\mathrm{C}$ & \\
\hline Tyto alba & $\mathrm{x}$ & & & & $\mathrm{x}$ & $\mathrm{x}$ & & & $\mathrm{R}$ & $\mathrm{C}$ & $\mathrm{R}$ & \\
\hline Megascops guatemalae & $\mathrm{x}$ & $\mathrm{x}$ & $\mathrm{x}$ & & & & & & $\mathrm{R}$ & $\mathrm{C}$ & $\mathrm{I}$ & \\
\hline Pulsatrix perspicillata & $\mathrm{x}$ & $\mathrm{x}$ & & & $\mathrm{x}$ & $\mathrm{x}$ & & & $\mathrm{R}$ & $\mathrm{C}$ & $\mathrm{R}$ & A \\
\hline Glaucidium brasilianum & $\mathrm{x}$ & & $\mathrm{x}$ & & & & & & $\mathrm{R}$ & $\mathrm{C}$ & $\mathrm{I}$ & \\
\hline Ciccaba virgata & $\mathrm{x}$ & & & & & & & & $\mathrm{R}$ & $\mathrm{C}$ & $\mathrm{R}$ & \\
\hline Nyctidromus albicollis & $\mathrm{x}$ & & $\mathrm{x}$ & & & & & & $\mathrm{R}$ & I & $\mathrm{R}$ & \\
\hline Phaethornis longirostris & $\mathrm{x}$ & & $\mathrm{x}$ & & & & & & $\mathrm{R}$ & $\mathrm{N}$ & $\mathrm{E}$ & \\
\hline Phaethornis striigularis & $\mathrm{x}$ & & & & & & & & $\mathrm{R}$ & $\mathrm{N}$ & I & \\
\hline Florisuga mellivora & $\mathrm{x}$ & $\mathrm{x}$ & & & & & & & $\mathrm{R}$ & $\mathrm{N}$ & $\mathrm{R}$ & \\
\hline Colibri thalassinus & $\mathrm{x}$ & & $\mathrm{x}$ & & & & & & $\mathrm{R}$ & $\mathrm{N}$ & $\mathrm{E}$ & \\
\hline Chlorostilbon canivetii & $\mathrm{x}$ & & $\mathrm{x}$ & & & & & & $\mathrm{R}$ & $\mathrm{N}$ & $\mathrm{C}$ & \\
\hline Amazilia beryllina & $\mathrm{x}$ & $\mathrm{x}$ & & & & & $\mathrm{x}$ & $\mathrm{x}$ & $\mathrm{R}$ & $\mathrm{N}$ & I & \\
\hline Amazilia tzacatl & $\mathrm{x}$ & & $\mathrm{x}$ & & & & $\mathrm{x}$ & $\mathrm{x}$ & $\mathrm{R}$ & $\mathrm{N}$ & $\mathrm{C}$ & \\
\hline Amazilia yucatanensis & $\mathrm{x}$ & & $\mathrm{x}$ & & & & & & $\mathrm{R}$ & $\mathrm{N}$ & $\mathrm{E}$ & \\
\hline Amazilia viridifrons & $\mathrm{x}$ & & $\mathrm{x}$ & & & & & & $\mathrm{R}$ & $\mathrm{N}$ & $\mathrm{C}$ & A \\
\hline Eupherusa eximia & $\mathrm{x}$ & & $\mathrm{x}$ & & & & & & $\mathrm{R}$ & $\mathrm{N}$ & $\mathrm{I}$ & \\
\hline Lamprolaima rhami & $\mathrm{x}$ & $\mathrm{x}$ & & & & & & & $\mathrm{R}$ & $\mathrm{N}$ & $\mathrm{R}$ & $\mathrm{A}$ \\
\hline Heliomaster longirostris & $\mathrm{x}$ & & $\mathrm{x}$ & & & & & & $\mathrm{R}$ & $\mathrm{N}$ & $\mathrm{R}$ & $\operatorname{Pr}$ \\
\hline Tilmatura dupontii & $\mathrm{x}$ & $\mathrm{x}$ & $\mathrm{x}$ & & & & & & $\mathrm{R}$ & $\mathrm{N}$ & I & A \\
\hline Archilochus colubris & $\mathrm{x}$ & & & & & & & & W & $\mathrm{N}$ & $\mathrm{R}$ & \\
\hline Trogon melanocephalus & $\mathrm{x}$ & $\mathrm{x}$ & & & & & & & $\mathrm{R}$ & IF & $\mathrm{C}$ & \\
\hline Trogon violaceus & $\mathrm{x}$ & $\mathrm{x}$ & & & & & & & $\mathrm{R}$ & IF & $\mathrm{R}$ & \\
\hline Momotus mexicanus & $\mathrm{x}$ & $\mathrm{x}$ & & $\mathrm{x}$ & & & & $\mathrm{x}$ & $\mathrm{R}$ & CIF & $\mathrm{C}$ & \\
\hline Momotus momota & $\mathrm{x}$ & $\mathrm{x}$ & & & & & & & $\mathrm{R}$ & CIF & $\mathrm{E}$ & \\
\hline Megaceryle alcyon & & & & & & & & $\mathrm{x}$ & W & $\mathrm{C}$ & $\mathrm{R}$ & \\
\hline Chloroceryle amazona & & & & & & & & $\mathrm{x}$ & $\mathrm{R}$ & $\mathrm{CI}$ & $\mathrm{R}$ & \\
\hline Aulacorhynchus prasinus & & $\mathrm{x}$ & $\mathrm{x}$ & & & & & & $\mathrm{R}$ & GF & $\mathrm{R}$ & $\operatorname{Pr}$ \\
\hline Ramphastos sulfuratus & & $\mathrm{x}$ & $\mathrm{x}$ & & & & & & $\mathrm{R}$ & GF & $\mathrm{R}$ & $\mathrm{A}$ \\
\hline Melanerpes aurifrons & $\mathrm{x}$ & & $\mathrm{x}$ & $\mathrm{x}$ & & & & & $\mathrm{R}$ & $\mathrm{IF}$ & $\mathrm{C}$ & \\
\hline Picoides scalaris & & & $\mathrm{x}$ & & & & & & $\mathrm{R}$ & I & $\mathrm{R}$ & \\
\hline Colaptes rubiginosus & $\mathrm{x}$ & & $\mathrm{x}$ & & & & & & $\mathrm{R}$ & I & $\mathrm{I}$ & \\
\hline Sclerurus guatemalensis & $\mathrm{x}$ & & & & & & & & $\mathrm{R}$ & I & $\mathrm{R}$ & $\operatorname{Pr}$ \\
\hline Synallaxis erythrothorax & $\mathrm{x}$ & & & & & & & & $\mathrm{R}$ & I & I & \\
\hline Automolus ochrolaemus & $\mathrm{x}$ & & $\mathrm{x}$ & & & & & & $\mathrm{R}$ & I & $\mathrm{R}$ & $\operatorname{Pr}$ \\
\hline Dendrocincla homochroa & $\mathrm{x}$ & & & & & & & & $\mathrm{R}$ & I & $\mathrm{R}$ & \\
\hline $\begin{array}{l}\text { Dendrocolaptes } \\
\text { sanctithomae }\end{array}$ & $\mathrm{x}$ & & & & & & & & $\mathrm{R}$ & I & $\mathrm{R}$ & \\
\hline Xiphorhynchus flavigaster & $\mathrm{x}$ & $\mathrm{x}$ & & & & & & & $\mathrm{R}$ & I & $\mathrm{E}$ & \\
\hline Lepidocolaptes souleyetii & $\mathrm{x}$ & $\mathrm{x}$ & $\mathrm{x}$ & & & & & & $\mathrm{R}$ & I & $\mathrm{E}$ & \\
\hline Taraba major & $\mathrm{x}$ & & $\mathrm{x}$ & & & & $\mathrm{x}$ & & $\mathrm{R}$ & I & I & $\operatorname{Pr}$ \\
\hline Thamnophilus doliatus & $\mathrm{x}$ & & $\mathrm{x}$ & & & $\mathrm{x}$ & $\mathrm{x}$ & & $\mathrm{R}$ & I & $\mathrm{C}$ & \\
\hline Cercomacra tyrannina & $\mathrm{x}$ & & $\mathrm{x}$ & & & & & & $\mathrm{R}$ & I & I & \\
\hline
\end{tabular}


ANEXO 1 (Continuación)

Especies de aves del área de estudio en la Depresión Central de Chiapas, México

APPENDIX 1 (Continued)

Bird species in the study area, Central Depression of Chiapas, Mexico

\begin{tabular}{|c|c|c|c|c|c|c|c|c|c|c|c|c|}
\hline \multirow[b]{2}{*}{ Especies } & \multicolumn{8}{|c|}{ Hábitats } & \multirow{2}{*}{$\begin{array}{l}\text { Estatus } \\
\text { migratorio }\end{array}$} & \multirow[b]{2}{*}{ Dieta } & \multirow{2}{*}{$\begin{array}{l}\text { Abundancia } \\
\text { relativa }\end{array}$} & \multirow{2}{*}{$\begin{array}{c}\text { Estatus } \\
\text { en la } \\
\text { NOM-059 }\end{array}$} \\
\hline & BTC & BTM & Vs & $\mathrm{BE}$ & $\mathrm{Pp}$ & $\mathrm{C}$ & $\mathrm{Cv}$ & BG & & & & \\
\hline Ornithion semiflavum & $\mathrm{x}$ & & & & & & & & $\mathrm{R}$ & I & $\mathrm{R}$ & $\operatorname{Pr}$ \\
\hline Camptostoma imberbe & $\mathrm{x}$ & & $\mathrm{x}$ & & & & $\mathrm{x}$ & & $\mathrm{R}$ & I & I & \\
\hline Myiopagis viridicata & $\mathrm{x}$ & & $\mathrm{x}$ & & & & & & $\mathrm{R}$ & I & $\mathrm{E}$ & \\
\hline Elaenia flavogaster & $\mathrm{x}$ & & $\mathrm{x}$ & & & & & & $\mathrm{R}$ & IF & $\mathrm{R}$ & \\
\hline $\begin{array}{l}\text { Leptopogon } \\
\text { amaurocephalus }\end{array}$ & $\mathrm{x}$ & & $\mathrm{x}$ & & & & & & $\mathrm{R}$ & I & I & \\
\hline Oncostoma cinereigulare & $\mathrm{x}$ & & & & & & & & $\mathrm{R}$ & I & $\mathrm{R}$ & \\
\hline Poecilotriccus sylvia & $\mathrm{x}$ & $\mathrm{x}$ & & & & & & & $\mathrm{R}$ & I & $\mathrm{E}$ & \\
\hline Rhynchocyclus brevirostris & $\mathrm{x}$ & & $\mathrm{x}$ & & & & $\mathrm{x}$ & & $\mathrm{R}$ & I & $\mathrm{I}$ & \\
\hline Contopus pertinax & $\mathrm{x}$ & & $\mathrm{x}$ & $\mathrm{x}$ & $\mathrm{x}$ & & $\mathrm{x}$ & $\mathrm{x}$ & $\mathrm{R}$ & I & $\mathrm{R}$ & \\
\hline Contopus virens & $\mathrm{x}$ & & $\mathrm{x}$ & & & & & & $\mathrm{T}$ & I & I & \\
\hline Contopus cinereus & $\mathrm{x}$ & & $\mathrm{x}$ & $\mathrm{x}$ & $\mathrm{x}$ & & $\mathrm{x}$ & $\mathrm{x}$ & $\mathrm{R}$ & I & I & \\
\hline Empidonax virescens & $\mathrm{x}$ & & $\mathrm{x}$ & & & & & & $\mathrm{T}$ & I & $\mathrm{R}$ & \\
\hline Empidonax traillii & $\mathrm{x}$ & & $\mathrm{x}$ & & & & & & $\mathrm{T}$ & IF & I & \\
\hline Empidonax albigularis & $\mathrm{x}$ & & $\mathrm{x}$ & $\mathrm{x}$ & & & $\mathrm{x}$ & $\mathrm{x}$ & W & IF & $\mathrm{E}$ & \\
\hline Sayornis nigricans & $\mathrm{x}$ & & & & & & & $\mathrm{x}$ & $\mathrm{R}$ & $\mathrm{I}$ & $\mathrm{R}$ & \\
\hline Pyrocephalus rubinus & & & & & $\mathrm{x}$ & & $\mathrm{x}$ & & $\mathrm{R}$ & I & $\mathrm{I}$ & \\
\hline Rhytipterna holerythra & $\mathrm{x}$ & & $\mathrm{x}$ & & & & & & $\mathrm{R}$ & $\mathrm{I}$ & I & \\
\hline Myiarchus tuberculifer & $\mathrm{x}$ & & & & $\mathrm{x}$ & & $\mathrm{x}$ & & $\mathrm{R}$ & IF & $\mathrm{R}$ & \\
\hline Myiarchus tyrannulus & $\mathrm{x}$ & $\mathrm{x}$ & $\mathrm{x}$ & $\mathrm{x}$ & $\mathrm{x}$ & & $\mathrm{x}$ & $\mathrm{x}$ & $\mathrm{R}$ & IF & $\mathrm{C}$ & \\
\hline Pitangus sulphuratus & $\mathrm{x}$ & $\mathrm{x}$ & $\mathrm{x}$ & $\mathrm{x}$ & $\mathrm{x}$ & & $\mathrm{x}$ & $\mathrm{x}$ & $\mathrm{R}$ & CIF & $\mathrm{C}$ & \\
\hline Megarhynchus pitangua & $\mathrm{x}$ & $\mathrm{x}$ & $\mathrm{x}$ & & & & $\mathrm{x}$ & $\mathrm{x}$ & $\mathrm{R}$ & CIF & $\mathrm{C}$ & \\
\hline Myiozetetes similis & $\mathrm{x}$ & $\mathrm{x}$ & $\mathrm{x}$ & $\mathrm{x}$ & $\mathrm{x}$ & & $\mathrm{x}$ & $\mathrm{x}$ & $\mathrm{R}$ & IF & $\mathrm{C}$ & \\
\hline Myiodynastes luteiventris & $\mathrm{x}$ & $\mathrm{x}$ & $\mathrm{x}$ & & & & $\mathrm{x}$ & $\mathrm{x}$ & W & IF & $\mathrm{E}$ & \\
\hline Legatus leucophaius & & $\mathrm{x}$ & & & & & & $\mathrm{x}$ & $\mathrm{S}$ & $\mathrm{I}$ & $\mathrm{R}$ & \\
\hline Tyrannus melancholicus & $\mathrm{x}$ & & $\mathrm{x}$ & $\mathrm{x}$ & $\mathrm{x}$ & & $\mathrm{x}$ & $\mathrm{x}$ & $\mathrm{R}$ & IF & $\mathrm{C}$ & \\
\hline Tyrannus vociferans & $\mathrm{x}$ & & & & $\mathrm{x}$ & & $\mathrm{x}$ & & $\mathrm{W}$ & IF & $\mathrm{E}$ & \\
\hline Tyrannus verticalis & & & $\mathrm{x}$ & & $\mathrm{x}$ & & $\mathrm{x}$ & & W & IF & $\mathrm{R}$ & \\
\hline Tyrannus tyrannus & & & $\mathrm{x}$ & & $\mathrm{x}$ & $\mathrm{x}$ & & & $\mathrm{T}$ & I & $\mathrm{R}$ & \\
\hline Tyrannus forficatus & & & $\mathrm{x}$ & & & & $\mathrm{x}$ & & W & IF & I & \\
\hline Pachyramphus aglaiae & $\mathrm{x}$ & & $\mathrm{x}$ & & & & & & $\mathrm{R}$ & IF & I & \\
\hline Tityra semifasciata & $\mathrm{x}$ & & $\mathrm{x}$ & & & & & & $\mathrm{R}$ & IF & $\mathrm{E}$ & \\
\hline Vireo griseus & $\mathrm{x}$ & & $\mathrm{x}$ & & & & $\mathrm{x}$ & & W & IF & I & \\
\hline Vireo bellii & $\mathrm{x}$ & & & & & & $\mathrm{x}$ & & $\mathrm{T}$ & I & $\mathrm{R}$ & \\
\hline Vireo solitarius & $\mathrm{x}$ & & $\mathrm{x}$ & $\mathrm{x}$ & & & $\mathrm{x}$ & $\mathrm{x}$ & W & IF & $\mathrm{E}$ & \\
\hline Vireo huttoni & $\mathrm{x}$ & & & & & & $\mathrm{x}$ & & $\mathrm{R}$ & I & $\mathrm{R}$ & \\
\hline Vireo gilvus & $\mathrm{x}$ & & $\mathrm{x}$ & $\mathrm{x}$ & & & $\mathrm{x}$ & & W & IF & $\mathrm{E}$ & \\
\hline Vireo leucophrys & $\mathrm{x}$ & & & & & & & & $\mathrm{R}$ & IF & $\mathrm{R}$ & \\
\hline Vireo philadelphicus & $\mathrm{x}$ & & & & & & $\mathrm{x}$ & & $\mathrm{W}$ & I & I & \\
\hline Vireo olivaceus & $\mathrm{x}$ & & $\mathrm{x}$ & $\mathrm{x}$ & & & $\mathrm{x}$ & $\mathrm{x}$ & $\mathrm{T}$ & IF & $\mathrm{I}$ & \\
\hline Vireo flavoviridis & $\mathrm{x}$ & $\mathrm{x}$ & & & & & & & W & IF & $\mathrm{E}$ & \\
\hline Hylophilus decurtatus & $\mathrm{x}$ & & $\mathrm{x}$ & & & & $\mathrm{x}$ & & $\mathrm{R}$ & I & I & \\
\hline Cyclarhis gujanensis & $\mathrm{x}$ & $\mathrm{x}$ & $\mathrm{x}$ & & & & $\mathrm{x}$ & & $\mathrm{R}$ & IF & $\mathrm{E}$ & \\
\hline Calocitta formosa & $\mathrm{x}$ & $\mathrm{x}$ & $\mathrm{x}$ & $\mathrm{x}$ & & $\mathrm{x}$ & $x$ & $\mathrm{x}$ & $\mathrm{R}$ & $\mathrm{O}$ & A & \\
\hline
\end{tabular}


ANEXO 1 (Continuación)

Especies de aves del área de estudio en la Depresión Central de Chiapas, México

APPENDIX 1 (Continued)

Bird species in the study area, Central Depression of Chiapas, Mexico

\begin{tabular}{|c|c|c|c|c|c|c|c|c|c|c|c|c|}
\hline \multirow[b]{2}{*}{ Especies } & \multicolumn{8}{|c|}{ Hábitats } & \multirow{2}{*}{$\begin{array}{c}\text { Estatus } \\
\text { migratorio }\end{array}$} & \multirow[b]{2}{*}{ Dieta } & \multirow{2}{*}{$\begin{array}{c}\text { Abundancia } \\
\text { relativa }\end{array}$} & \multirow{2}{*}{$\begin{array}{c}\text { Estatus } \\
\text { en la } \\
\text { NOM-059 }\end{array}$} \\
\hline & BTC & BTM & Vs & $\mathrm{BE}$ & $\mathrm{Pp}$ & $\mathrm{C}$ & $\mathrm{Cv}$ & BG & & & & \\
\hline Cyanocorax yncas & $\mathrm{x}$ & $\mathrm{x}$ & $\mathrm{x}$ & $\mathrm{x}$ & & & $\mathrm{x}$ & & $\mathrm{R}$ & $\mathrm{O}$ & $\mathrm{C}$ & \\
\hline Cyanocorax morio & $\mathrm{x}$ & $\mathrm{x}$ & $\mathrm{x}$ & $\mathrm{x}$ & & & & & $\mathrm{R}$ & $\mathrm{O}$ & A & \\
\hline Catherpes mexicanus & $\mathrm{x}$ & $\mathrm{x}$ & & & & & & $\mathrm{x}$ & $\mathrm{R}$ & $\mathrm{I}$ & $\mathrm{E}$ & \\
\hline Thryothorus maculipectus & $\mathrm{x}$ & & $\mathrm{x}$ & $\mathrm{x}$ & & & & & $\mathrm{R}$ & I & $\mathrm{C}$ & \\
\hline Thryothorus pleurostictus & $\mathrm{x}$ & $\mathrm{x}$ & $\mathrm{x}$ & $\mathrm{x}$ & & $\mathrm{x}$ & & & $\mathrm{R}$ & I & $\mathrm{C}$ & \\
\hline Thryothorus modestus & $\mathrm{x}$ & & $\mathrm{x}$ & & & $\mathrm{x}$ & & & $\mathrm{R}$ & I & $\mathrm{E}$ & \\
\hline Troglodytes aedon & $\mathrm{x}$ & & & & & & & & $\mathrm{R}$ & I & $\mathrm{I}$ & \\
\hline Cistothorus platensis & $\mathrm{x}$ & & & & & & & & $\mathrm{R}$ & I & $\mathrm{R}$ & \\
\hline Uropsila leucogastra & $\mathrm{x}$ & $\mathrm{x}$ & & & & & & & $\mathrm{R}$ & I & I & \\
\hline Henicorchia leucophrys & $\mathrm{x}$ & & $\mathrm{x}$ & & & & & & $\mathrm{R}$ & I & I & \\
\hline Microcerculus marginatus & & $\mathrm{x}$ & & & & & & & $\mathrm{R}$ & I & $\mathrm{R}$ & \\
\hline Ramphocaenus melanurus & $\mathrm{x}$ & & $\mathrm{x}$ & & & & & & $\mathrm{R}$ & $\mathrm{I}$ & $\mathrm{R}$ & \\
\hline Polioptila caerulea & $\mathrm{x}$ & & $\mathrm{x}$ & $\mathrm{x}$ & & & $\mathrm{x}$ & $\mathrm{x}$ & $\mathrm{R}$ & I & $\mathrm{C}$ & \\
\hline Polioptila plumbea & $\mathrm{x}$ & $\mathrm{x}$ & $\mathrm{x}$ & $\mathrm{x}$ & & & $\mathrm{x}$ & & $\mathrm{R}$ & $\mathrm{I}$ & $\mathrm{E}$ & $\operatorname{Pr}$ \\
\hline Catharus aurantiirostris & $\mathrm{x}$ & & & & & & & & $\mathrm{R}$ & IF & $\mathrm{R}$ & \\
\hline Catharus minimus & $\mathrm{x}$ & & & & & & & & $\mathrm{T}$ & IF & $\mathrm{R}$ & \\
\hline Catharus ustulatus & $\mathrm{x}$ & $\mathrm{x}$ & & & & & & & W & IF & I & \\
\hline Hylocichla mustelina & & $\mathrm{x}$ & & & & & & & $\mathrm{T}$ & IF & $\mathrm{I}$ & \\
\hline Turdus grayi & $\mathrm{x}$ & $\mathrm{x}$ & $\mathrm{x}$ & $\mathrm{x}$ & & & $\mathrm{x}$ & $\mathrm{x}$ & $\mathrm{R}$ & IF & $\mathrm{C}$ & \\
\hline Dumetella carolinensis & $\mathrm{x}$ & & $\mathrm{x}$ & & & & & & W & IF & I & \\
\hline Mimus gilvus & & & & & & $\mathrm{x}$ & $\mathrm{x}$ & & $\mathrm{R}$ & IF & I & \\
\hline Bombycilla cedrorum & $\mathrm{x}$ & & $\mathrm{x}$ & $\mathrm{x}$ & & & $\mathrm{x}$ & & W & IF & I & \\
\hline Vermivora pinus & $\mathrm{x}$ & & & & & & & & W & I & $\mathrm{R}$ & \\
\hline Vermivora celata & $\mathrm{x}$ & & & & & & & & W & IF & $\mathrm{R}$ & \\
\hline Vermivora ruficapilla & $\mathrm{x}$ & & $\mathrm{x}$ & $\mathrm{x}$ & & & $\mathrm{x}$ & $\mathrm{x}$ & W & IF & $\mathrm{E}$ & \\
\hline Parula americana & $\mathrm{x}$ & & & & & & $\mathrm{x}$ & & $\mathrm{W}$ & I & I & \\
\hline Parula pitiayumi & $\mathrm{x}$ & & & & & & $\mathrm{x}$ & & $\mathrm{R}$ & I & I & \\
\hline Dendroica petechia & $\mathrm{x}$ & & & $\mathrm{x}$ & & & $\mathrm{x}$ & & W & IF & $\mathrm{E}$ & \\
\hline Dendroica pensylvanica & $\mathrm{x}$ & & & & & & & & W & IF & $\mathrm{R}$ & \\
\hline Dendroica magnolia & $\mathrm{x}$ & & $\mathrm{x}$ & $\mathrm{x}$ & & & $\mathrm{x}$ & & W & IF & $\mathrm{E}$ & \\
\hline Dendroica coronata & & $\mathrm{x}$ & & & & & & & W & IF & $\mathrm{R}$ & \\
\hline Dendroica virens & $\mathrm{x}$ & $\mathrm{x}$ & $\mathrm{x}$ & $\mathrm{x}$ & & & $\mathrm{x}$ & $\mathrm{x}$ & W & IF & $\mathrm{E}$ & \\
\hline Dendroica townsendi & $\mathrm{x}$ & & & & & & $\mathrm{x}$ & & W & IF & $\mathrm{R}$ & \\
\hline Dendroica occidentalis & $\mathrm{x}$ & & $\mathrm{x}$ & & & & $\mathrm{x}$ & & W & IF & I & \\
\hline Dendroica fusca & $\mathrm{x}$ & & & & & & & & $\mathrm{T}$ & I & $\mathrm{R}$ & \\
\hline Dendroica graciae & $\mathrm{x}$ & $\mathrm{x}$ & & $\mathrm{x}$ & & & $\mathrm{x}$ & & $\mathrm{R}$ & IF & $\mathrm{E}$ & \\
\hline Mniotilta varia & $\mathrm{x}$ & $\mathrm{x}$ & $\mathrm{x}$ & $\mathrm{x}$ & & & $\mathrm{x}$ & $\mathrm{x}$ & W & I & $\mathrm{E}$ & \\
\hline Protonotaria citrea & $\mathrm{x}$ & & & & & & & & $\mathrm{T}$ & I & $\mathrm{R}$ & \\
\hline Helmitheros vermivorum & & $\mathrm{x}$ & & & & & & & W & I & $\mathrm{R}$ & \\
\hline Seiurus aurocapilla & $\mathrm{x}$ & $\mathrm{x}$ & $\mathrm{x}$ & & & & & & W & I & $\mathrm{E}$ & \\
\hline Oporornis formosus & $\mathrm{x}$ & & & & & & & & $\mathrm{T}$ & I & $\mathrm{R}$ & \\
\hline Oporornis tolmiei & $\mathrm{x}$ & & & & & & & & W & I & I & A \\
\hline Geothlypis trichas & & & & & $\mathrm{x}$ & $\mathrm{x}$ & & & W & I & $\mathrm{E}$ & \\
\hline Geothlypis poliocephala & & & & & $\mathrm{x}$ & $\mathrm{x}$ & & & $\mathrm{R}$ & I & $\mathrm{E}$ & \\
\hline
\end{tabular}


ANEXO 1 (Continuación)

Especies de aves del área de estudio en la Depresión Central de Chiapas, México

APPENDIX 1 (Continued)

Bird species in the study area, Central Depression of Chiapas, Mexico

\begin{tabular}{|c|c|c|c|c|c|c|c|c|c|c|c|c|}
\hline \multirow{2}{*}{ Especies } & \multicolumn{8}{|c|}{ Hábitats } & \multirow{2}{*}{$\begin{array}{c}\text { Estatus } \\
\text { migratorio }\end{array}$} & \multirow{2}{*}{ Dieta } & \multirow{2}{*}{$\begin{array}{l}\text { Abundancia } \\
\text { relativa }\end{array}$} & \multirow{2}{*}{$\begin{array}{c}\text { Estatus } \\
\text { en la } \\
\text { NOM-059 }\end{array}$} \\
\hline & BTC & BTM & Vs & $\mathrm{BE}$ & $\mathrm{Pp}$ & $\mathrm{C}$ & $\mathrm{Cv}$ & BG & & & & \\
\hline Wilsonia citrina & $\mathrm{x}$ & & $\mathrm{x}$ & & & & $\mathrm{x}$ & & W & $\mathrm{I}$ & $\mathrm{R}$ & \\
\hline Wilsonia pusilla & $\mathrm{x}$ & $\mathrm{x}$ & $\mathrm{x}$ & $\mathrm{x}$ & & & $\mathrm{x}$ & $\mathrm{x}$ & W & IF & $\mathrm{E}$ & \\
\hline Wilsonia canadensis & $\mathrm{x}$ & & $\mathrm{x}$ & & & & $\mathrm{x}$ & & $\mathrm{T}$ & IF & I & \\
\hline Euthlypis lachrymosa & $\mathrm{x}$ & $\mathrm{x}$ & $\mathrm{x}$ & & & & & $\mathrm{x}$ & $\mathrm{R}$ & I & I & $\operatorname{Pr}$ \\
\hline Basileuterus rufifrons & $\mathrm{x}$ & & $\mathrm{x}$ & & & & $\mathrm{x}$ & & $\mathrm{R}$ & $\mathrm{I}$ & I & \\
\hline Icteria virens & & & $\mathrm{x}$ & & & & $\mathrm{x}$ & & $\mathrm{W}$ & I & I & \\
\hline Granatellus venustus & $\mathrm{x}$ & & & & & & & & $\mathrm{R}$ & I & $\mathrm{I}$ & \\
\hline Granatellus sallaei & $\mathrm{x}$ & & & & & & & & $\mathrm{R}$ & $\mathrm{I}$ & $\mathrm{R}$ & \\
\hline Eucometis penicillata & $\mathrm{x}$ & & & & & & & & $\mathrm{R}$ & IF & $\mathrm{R}$ & \\
\hline Habia rubica & $\mathrm{x}$ & & $\mathrm{x}$ & & & & & & $\mathrm{R}$ & IF & I & \\
\hline Piranga rubra & $\mathrm{x}$ & $\mathrm{x}$ & $\mathrm{x}$ & & & & & & W & IF & $\mathrm{I}$ & \\
\hline Piranga ludoviciana & $\mathrm{x}$ & & $\mathrm{x}$ & $\mathrm{x}$ & & & $\mathrm{x}$ & & W & IF & $\mathrm{E}$ & \\
\hline Thraupis episcopus & & & $\mathrm{x}$ & & & & $\mathrm{x}$ & & $\mathrm{R}$ & IF & $\mathrm{R}$ & \\
\hline Thraupis abbas & $\mathrm{x}$ & $\mathrm{x}$ & & $\mathrm{x}$ & & & $\mathrm{x}$ & $\mathrm{x}$ & $\mathrm{R}$ & IF & $\mathrm{E}$ & \\
\hline Cyanerpes cyaneus & $\mathrm{x}$ & $\mathrm{x}$ & & & & & & & $\mathrm{R}$ & $\mathrm{IN}$ & $\mathrm{R}$ & \\
\hline Volatinia jacarina & & & & & $\mathrm{x}$ & $\mathrm{x}$ & & & $\mathrm{R}$ & G & $\mathrm{C}$ & \\
\hline Sporophila torqueola & & & & & $\mathrm{x}$ & $\mathrm{x}$ & & & $\mathrm{R}$ & G & $\mathrm{E}$ & \\
\hline Oryzoborus funereus & & & & & $\mathrm{x}$ & $\mathrm{x}$ & & & $\mathrm{R}$ & G & $\mathrm{E}$ & \\
\hline Tiaris olivaceus & & & $\mathrm{x}$ & & $\mathrm{x}$ & $\mathrm{x}$ & $\mathrm{x}$ & & $\mathrm{R}$ & G & $\mathrm{R}$ & \\
\hline Arremonops rufivirgatus & $\mathrm{x}$ & $\mathrm{x}$ & $\mathrm{x}$ & & $\mathrm{x}$ & $\mathrm{x}$ & $\mathrm{x}$ & & $\mathrm{R}$ & GI & $\mathrm{C}$ & \\
\hline Aimophila botterii & & & & & $\mathrm{x}$ & $\mathrm{x}$ & & & $\mathrm{R}$ & $\mathrm{G}$ & $\mathrm{I}$ & \\
\hline Aimophila rufescens & & & & & $\mathrm{x}$ & $\mathrm{x}$ & & & $\mathrm{R}$ & GI & $\mathrm{E}$ & \\
\hline Saltator coerulescens & $\mathrm{x}$ & & $\mathrm{x}$ & & & & $\mathrm{x}$ & & $\mathrm{R}$ & IF & I & \\
\hline Saltator atriceps & $\mathrm{x}$ & & $\mathrm{x}$ & & & & $\mathrm{x}$ & & $\mathrm{R}$ & IF & $\mathrm{E}$ & \\
\hline Pheucticus chrysopeplus & $\mathrm{x}$ & $\mathrm{x}$ & $\mathrm{x}$ & $\mathrm{x}$ & & & & & $\mathrm{R}$ & IF & $\mathrm{E}$ & \\
\hline Pheucticus ludovicianus & & & $\mathrm{x}$ & $\mathrm{x}$ & & & & & W & IF & $\mathrm{R}$ & \\
\hline Cyanocompsa parellina & $\mathrm{x}$ & & $\mathrm{x}$ & & & $\mathrm{x}$ & & & $\mathrm{R}$ & G & $\mathrm{E}$ & \\
\hline Passerina caerulea & & & & & $\mathrm{x}$ & $\mathrm{x}$ & $\mathrm{x}$ & & $\mathrm{R}$ & GI & $\mathrm{C}$ & \\
\hline Passerina cyanea & $\mathrm{x}$ & & & & $\mathrm{x}$ & $\mathrm{x}$ & & & W & GI & $\mathrm{E}$ & \\
\hline Passerina versicolor & $\mathrm{x}$ & & $\mathrm{x}$ & & $\mathrm{x}$ & $\mathrm{x}$ & & & $\mathrm{R}$ & GIF & $\mathrm{E}$ & \\
\hline Passerina ciris & & & $\mathrm{x}$ & & & $\mathrm{x}$ & & & W & GI & $\mathrm{I}$ & \\
\hline Sturnella magna & & & & & $\mathrm{x}$ & $\mathrm{x}$ & & & $\mathrm{R}$ & GI & $\mathrm{R}$ & \\
\hline Dives dives & $\mathrm{x}$ & $\mathrm{x}$ & $\mathrm{x}$ & $\mathrm{x}$ & & & $\mathrm{x}$ & $\mathrm{x}$ & $\mathrm{R}$ & GIF & A & \\
\hline Quiscalus mexicanus & $\mathrm{x}$ & $\mathrm{x}$ & $\mathrm{x}$ & $\mathrm{x}$ & $\mathrm{x}$ & $\mathrm{x}$ & $\mathrm{x}$ & $\mathrm{x}$ & $\mathrm{R}$ & $\mathrm{O}$ & A & \\
\hline Molothrus aeneus & & & & $\mathrm{x}$ & $\mathrm{x}$ & $\mathrm{x}$ & $\mathrm{x}$ & & $\mathrm{R}$ & GIF & $\mathrm{C}$ & \\
\hline Icterus prosthemelas & $\mathrm{x}$ & & $\mathrm{x}$ & & & & $\mathrm{x}$ & & $\mathrm{R}$ & IF & $\mathrm{R}$ & \\
\hline Icterus wagleri & $\mathrm{x}$ & & $\mathrm{x}$ & & & & $\mathrm{x}$ & & $\mathrm{R}$ & IF & $\mathrm{E}$ & \\
\hline Icterus maculialatus & $\mathrm{x}$ & & & & & & & & $\mathrm{R}$ & IF & I & $\operatorname{Pr}$ \\
\hline Icterus spurius & & & $\mathrm{x}$ & & & $\mathrm{x}$ & $\mathrm{x}$ & & W & IF & $\mathrm{R}$ & \\
\hline Icterus mesomelas & $\mathrm{x}$ & & $\mathrm{x}$ & & & & $\mathrm{x}$ & & $\mathrm{R}$ & IF & I & \\
\hline Icterus pustulatus & $\mathrm{x}$ & $\mathrm{x}$ & $\mathrm{x}$ & $\mathrm{x}$ & & & $\mathrm{x}$ & $\mathrm{x}$ & $\mathrm{R}$ & IF & $\mathrm{C}$ & \\
\hline Icterus gularis & $\mathrm{x}$ & $\mathrm{x}$ & $\mathrm{x}$ & & & & $\mathrm{x}$ & $\mathrm{x}$ & $\mathrm{R}$ & IF & $\mathrm{C}$ & \\
\hline Icterus galbula & $\mathrm{x}$ & & & & & & $\mathrm{x}$ & & W & IF & I & \\
\hline Amblycercus holosericeus & $\mathrm{x}$ & $\mathrm{x}$ & & & & & & & $\mathrm{R}$ & IF & I & \\
\hline
\end{tabular}


ANEXO 1 (Continuación)

Especies de aves del área de estudio en la Depresión Central de Chiapas, México

APPENDIX 1 (Continued)

Bird species in the study area, Central Depression of Chiapas, Mexico

\begin{tabular}{|c|c|c|c|c|c|c|c|c|c|c|c|}
\hline \multirow[b]{2}{*}{ Especies } & \multicolumn{7}{|c|}{ Hábitats } & \multirow{2}{*}{$\begin{array}{l}\text { Estatus } \\
\text { migratorio }\end{array}$} & \multirow[b]{2}{*}{ Dieta } & \multirow{2}{*}{$\begin{array}{c}\text { Abundancia } \\
\text { relativa }\end{array}$} & \multirow{2}{*}{$\begin{array}{c}\text { Estatus } \\
\text { en la } \\
\text { NOM-059 }\end{array}$} \\
\hline & BTC & BTM & Vs & $\mathrm{BE}$ & $\mathrm{Pp}$ & $\mathrm{C}$ & BG & & & & \\
\hline Cacicus melanicterus & $\mathrm{x}$ & & & & & & & $\mathrm{T}$ & IF & $\mathrm{R}$ & \\
\hline Psarcolius wagleri & $\mathrm{x}$ & $\mathrm{x}$ & & & & & & $\mathrm{R}$ & GF & $\mathrm{R}$ & $\operatorname{Pr}$ \\
\hline Psarcolius montezuma & $\mathrm{x}$ & & $\mathrm{x}$ & & & & & $\mathrm{R}$ & GF & $\mathrm{R}$ & $\operatorname{Pr}$ \\
\hline Euphonia affinis & $\mathrm{x}$ & & $\mathrm{x}$ & & & & $\mathrm{x}$ & $\mathrm{R}$ & IF & $\mathrm{E}$ & \\
\hline Euphonia hirundinacea & $\mathrm{x}$ & $\mathrm{x}$ & $\mathrm{x}$ & $\mathrm{x}$ & & & $\mathrm{x}$ & $\mathrm{R}$ & IF & $\mathrm{E}$ & \\
\hline Carpodacus mexicanus & & & & & $\mathrm{x}$ & $\mathrm{x}$ & & $\mathrm{R}$ & G & $\mathrm{R}$ & \\
\hline Carduelis psaltria & & & $\mathrm{x}$ & $\mathrm{x}$ & $\mathrm{x}$ & $\mathrm{x}$ & $\mathrm{x}$ & $\mathrm{R}$ & GF & $\mathrm{E}$ & \\
\hline
\end{tabular}

Estatus migratorio (Estatus): R (residente), W (visitante de invierno), O (ocasional), T (transitoria), S (residente de verano). Abundancia (Abundancia): A (abundante), C (común), E (escasa), I (irregular), R (rara). Dieta/subdieta (Dieta): O (omnívoro), C (carnívoro), G (granívoro), F (frugívoro), I (insectívoro), N (nectarívoro). Hábitats: BTC (bosque tropical caducifolio), Pp (potrero/pastizal), Cv (cercos vivos), BG (bosque de galería), BTM (bosque tropical mediano), Vs (vegetación secundaria), C (zonas de cultivo), y BE (encinar tropical). Estatus en la NOM-059-SEMARNAT-2001 (SEMARNAT 2002): A (amenazada), Pr (bajo protección especial) y P (en peligro de extinción). 